\title{
Multifunctional Platform Based on Electrospun Nanofibers and Plasmonic Hydrogel: A Smart Nanostructured Pillow for Near- Infrared Light-Driven Biomedical Applications
}

\author{
Paweł Nakielski, ${ }^{\#}$ Sylwia Pawłowska, " Chiara Rinoldi, Yasamin Ziai, Luciano De Sio, Olga Urbanek, \\ Krzysztof Zembrzycki, Michał Pruchniewski, Massimiliano Lanzi, Elisabetta Salatelli, Antonella Calogero, \\ Tomasz A. Kowalewski, Alexander L. Yarin, and Filippo Pierini*
}

Cite This: ACS Appl. Mater. Interfaces 2020, 12, 54328-54342

Read Online

ABSTRACT: Multifunctional nanomaterials with the ability to respond to near-infrared (NIR) light stimulation are vital for the development of highly efficient biomedical nanoplatforms with a polytherapeutic approach. Inspired by the mesoglea structure of jellyfish bells, a biomimetic multifunctional nanostructured pillow with fast photothermal responsiveness for NIR light-controlled ondemand drug delivery is developed. We fabricate a nanoplatform with several hierarchical levels designed to generate a series of controlled, rapid, and reversible cascade-like structural changes upon NIR light irradiation. The mechanical contraction of the nanostructured platform, resulting from the increase of temper-

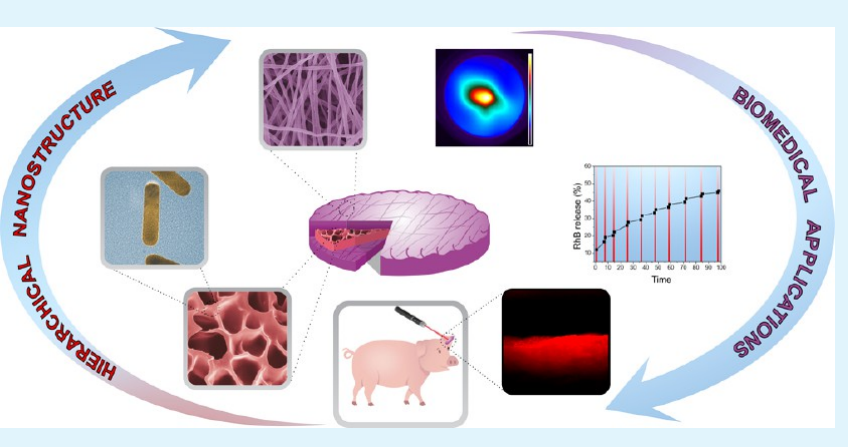
ature to $42{ }^{\circ} \mathrm{C}$ due to plasmonic hydrogel-light interaction, causes a rapid expulsion of water from the inner structure, passing through an electrospun membrane anchored onto the hydrogel core. The mutual effects of the rise in temperature and water flow stimulate the release of molecules from the nanofibers. To expand the potential applications of the biomimetic platform, the photothermal responsiveness to reach the typical temperature level for performing photothermal therapy (PTT) is designed. The on-demand drug model penetration into pig tissue demonstrates the efficiency of the nanostructured platform in the rapid and controlled release of molecules, while the high biocompatibility confirms the pillow potential for biomedical applications based on the NIR light-driven multitherapy strategy.

KEYWORDS: Bioinspired materials, NIR-light responsive nanomaterials, multifunctional platforms, electrospun nanofibers, plasmonic hydrogel, photothermal-based polytherapy, on-demand drug delivery

\section{INTRODUCTION}

Polymeric biomaterials offer great opportunities for increasing drug biodistribution in different biomedical applications (e.g., cancer or antibacterial therapy). ${ }^{1,2}$ However, traditional passive and active targeting strategies face many challenges, such as low tumor penetration in cancer treatment or low internalization of hydrophilic drugs in bacterial cells, such as in the case of antimicrobials. $^{3,4}$ Moreover, cancer cells develop different mechanisms to resist conventional monotherapy treatment (e.g., chemotherapy), while in the case of bacterial infections, multidrug-resistant bacteria become prevalent. This example confirms that there is an urgent need to develop multitherapies and on-demand treatments to improve drug delivery and bioavailability. ${ }^{5,6}$ In this context, several "smart" drug delivery systems are developed to obtain more effective treatments by providing novel characteristics and multiple properties that can respond to distinct environmental cues. ${ }^{7-9}$

Stimuli-responsive materials are used in many biomedical applications (e.g., tissue engineering, biosensing) and are considered potential candidates for pulsatile, site-specific, and externally stimulated On/Off drug release systems. ${ }^{10-12}$ Among this group of materials, hydrogels have attracted wide attention due to their tunable properties, which include mechanical strength, as well as structural, chemical, and biological responses to stimuli. ${ }^{11,13}$ One of the most studied stimuli-responsive hydrogels so far is a thermoresponsive polymer called poly $(\mathrm{N}$ isopropylacrylamide) (PNIPAAm).$^{14-16}$ The PNIPAAm phase transition from coil to globule appears when the temperature exceeds the typical low critical solution temperature (LCST) and is accompanied by a loss of polymer-water hydrogen bonds and formation of intra- and interchain hydrogen bonds between

Received: July 23, 2020

Accepted: November 16, 2020

Published: November 25, 2020 
a
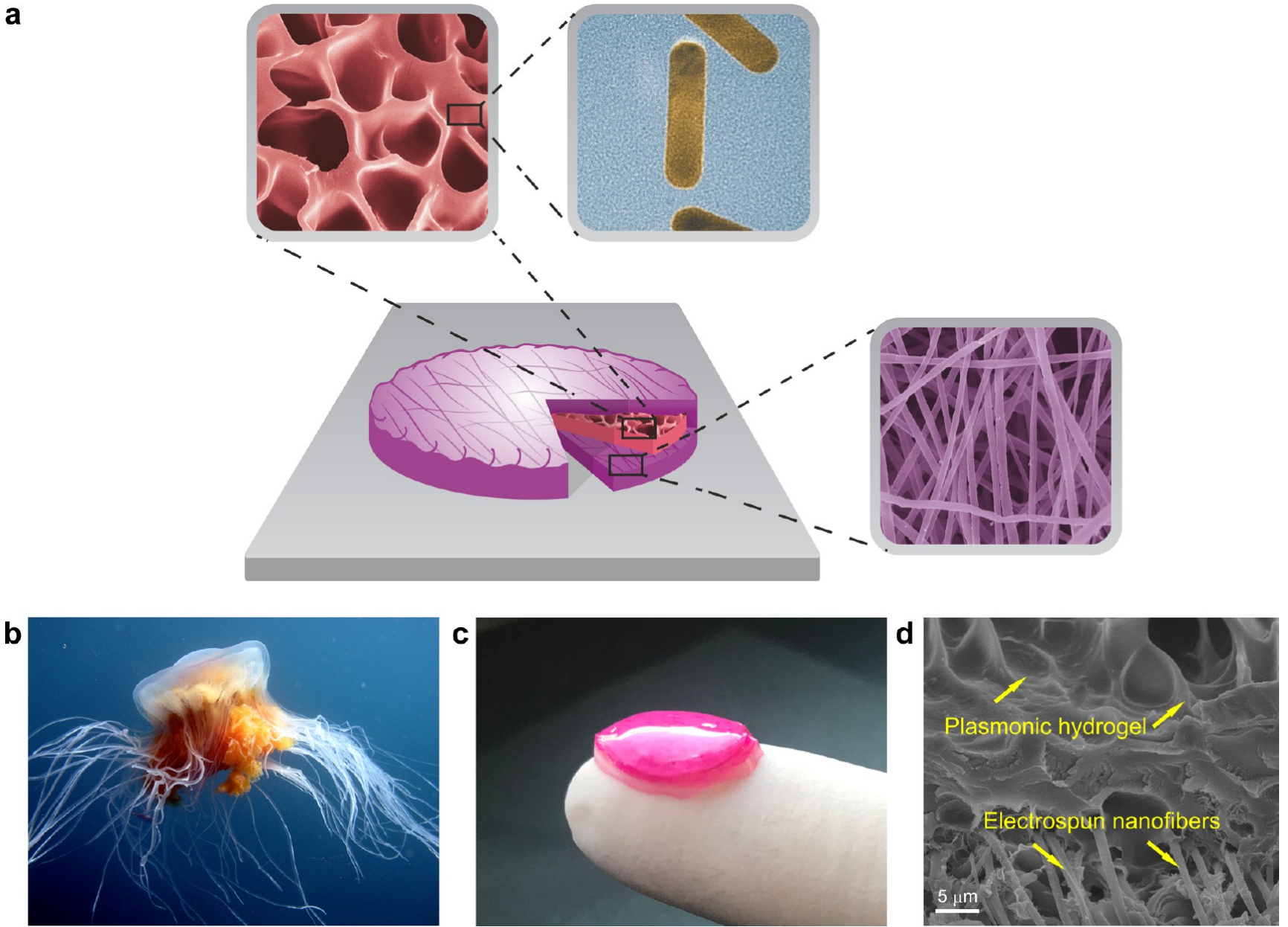

Figure 1. Bioinspired biomaterial design. (a) Scheme presenting the hierarchical nanostructuration of the developed platform: P(NIPAAm-coPNIPMAAm) hydrogel embedding gold nanorods (AuNRs) is encapsulated between two nanofibrous PLLA layers loaded with RhB. (b) Photography of a Phacellophora camtschatica jellyfish took near Gibraltar. Image courtesy of Prof. Stefano Piraino (University of Salento, Italy). (c) Photography of a bioinspired nanostructured pillow loaded with $\mathrm{RhB}$ showing the macroscopic appearance of the system. (d) Cross section of the fiber-hydrogel interface in the pillow inner structure, where P(NIPAAm-co-PNIPMAAm) hydrogel interpenetrates the PLLA nanofibrous layer pores, forming a biomimetic jellyfish mesoglea-like structure.

the amide groups, as well as dehydration of hydrophobic groups. ${ }^{17}$ At temperatures above LCST, PNIPAAm shrinks due to a sharp transition from the hydrophilic to the hydrophobic state. ${ }^{18}$ The phase transition above LCST causes the ejection of polymer-bound water. This process is fully reversible, and therefore, the hydrogel absorbs water and swells again when the temperature falls back below the LCST.

Because of the fact that PNIPAAm and other smart hydrogels can change their physical properties (e.g., volume) due to external stimuli, the incorporation of plasmonic nanoparticles (NPs) in the hydrogel matrix raises their level to a new class of biomaterials that are capable of responding to a stimulation in a cascade-like manner - plasmonic hydrogels. ${ }^{19,20}$ One of the benefits of embedding gold nanoparticles within the hydrogel matrix is that they can convert NIR light to heat due to the surface plasmon resonance (SPR) phenomenon, which generates localized photothermal effects. ${ }^{21}$ This increase in temperature can be cytotoxic for cancer cells or bacteria (photothermal therapy, PTT), thus also creating synergies with the drug action. ${ }^{22,23}$ More importantly, the generated heat can be used to locally increase the temperature above the system's LCST, trigger physical changes in the hydrogel (e.g., shrinking), and tune the mechanical properties.
An additional building block of our "smart" drug delivery systems is the nanofibrous nonwoven fabric. Recently, electrospun polymeric nanofibers have proven to be an attractive candidate for tissue engineering and drug delivery system applications. $^{24-27}$ The high loading capacity, simultaneous delivery of different molecules, and cost-effectiveness are the most attractive features of electrospun matrix use in drug delivery. Depending on several parameters affecting drugpolymer intermolecular forces, it is possible to modulate the drug release in such a way that the release process can take up to several weeks. ${ }^{28-30}$ With conventional electrospun systems, an On/Off drug delivery, which is a vital step for the development of an ideal on-demand pulsatile system, is not possible. On the one hand, most nanofibrous drug delivery biomaterials are still used unaccompanied, in a stand-alone system; therefore, it is necessary to properly design the materials' properties in order to obtain the desired release profile. ${ }^{28,31,32}$ Moreover, it is often reported that systems with high surface-to-volume ratios burst the drug from the surface of nanofibers, leading to unwanted therapeutic effects (e.g., drug toxicity). ${ }^{33}$ One of the solutions to this problem, as suggested in this paper, is the use of a polymer with strong polymer-drug interactions combined with a stimuliresponsive plasmonic hydrogel, which can increase the drug 

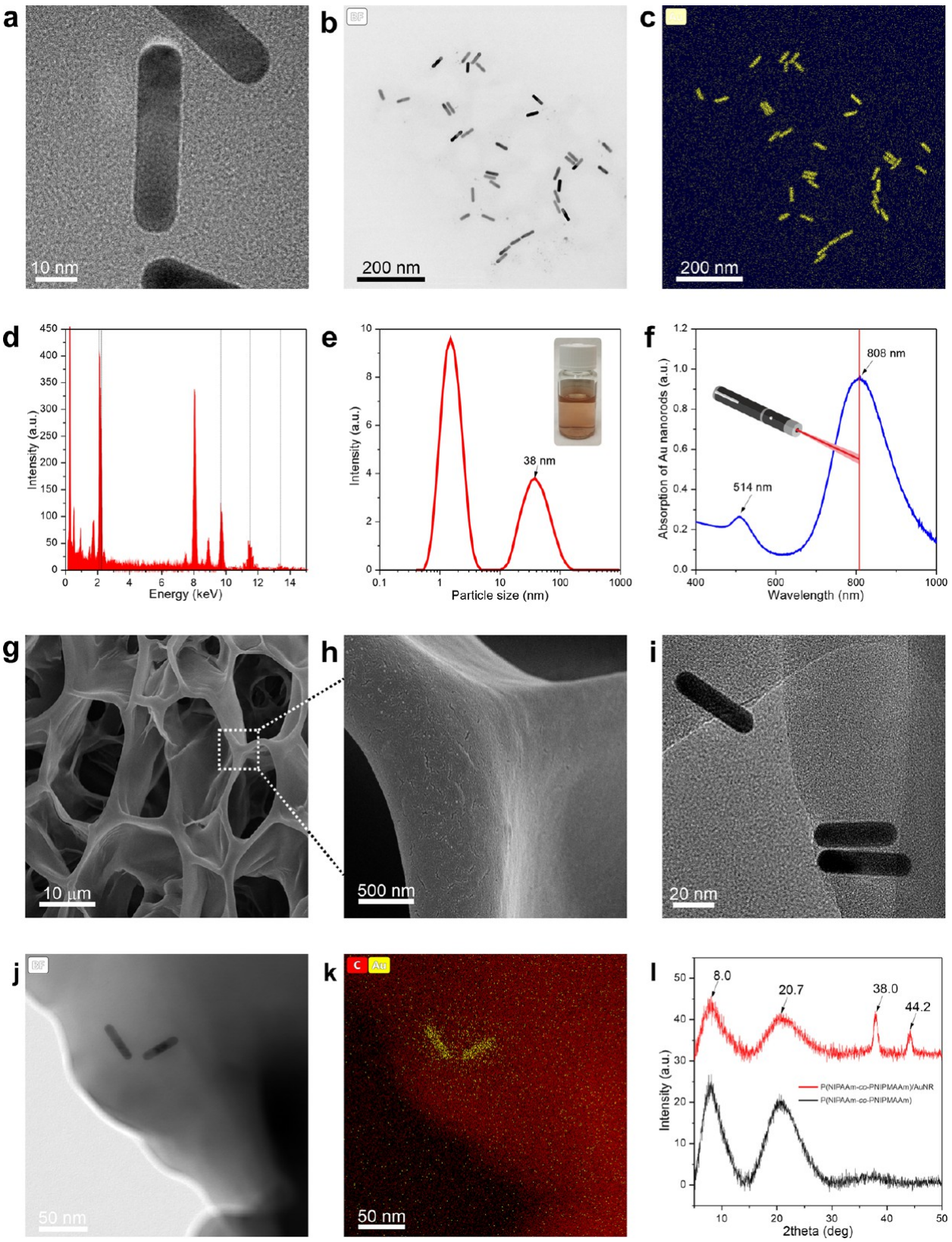

Figure 2. Characterization of gold nanorods and nanostructured P(NIPAAm-co-PNIPMAAm)/AuNR hydrogel. (a) High-resolution TEM image showing the crystalline structure of a single AuNR. (b) TEM image presenting gold nanorods' size and distribution in the hydrogel precursor. The average length was $42 \pm 3.5 \mathrm{~nm}$, while the width was $10 \pm 1.1 \mathrm{~nm}$. (c) TEM and EDX elemental mapping images confirming the presence of gold nanorods. (d) EDX spectrum of gold nanorods (vertical lines indicate the typical peaks of gold). (e) DLS plot showing the hydrodynamic radius of AuNR with an average hydrodynamic size of approximately $38 \mathrm{~nm}$ and an additional peak connected to the nanorod rotational diffusion. (f) UV-vis spectrum presenting the high absorption peaks of AuNR at 514 and $810 \mathrm{~nm}$. (g) SEM micrograph presenting freeze-dried nanostructured hydrogel. (h) High magnification of the hydrogel cell wall with no visible gold nanorods protruding from the hydrogel surface. (i) TEM imaging presenting AuNR embedded in the hydrogel. ( $\mathrm{j}$ ) TEM image presenting gold nanorod distribution in the hydrogel. (k) TEM and EDX elemental mapping images of gold nanorods embedded in the hydrogel. (1) WAXS diffractograms of P(NIPAAm-co-PNIPMAAm) control and P(NIPAAm-co-PNIPMAAm)/ AuNR highlight the two characteristic peaks of AuNRs at 38.05 and $44.25^{\circ}$ visible in the plasmonic hydrogel plot.

release rate on demand. By converting light to heat, these cascade systems can drastically enhance the drug release by increasing their kinetics and simultaneously inducing the hydrogel phase transition, thus leading to water expulsion and driving drug transport.

Here, we propose a bioinspired system, made of a poly(Llactide)-Rhodamine B-loaded nanofibrous material (PLLA$\mathrm{RhB}$ ), which encapsulates $\mathrm{P}$ (NIPAAm-co-NIPMAAm) hydrogel containing gold nanorods (AuNRs). Our stimuli-responsive material has structures that are similar to their natural counterparts, found in animals such as jellyfish or hydra. ${ }^{34-36}$ Mesoglea is a functional structure present in jellyfish bells, made of a hydrogel surrounded by a polymer nanofiber, which is a highly hydrated fibrous matter that works as a hydrostatic skeleton and can change its volume due to external stimuli, working similarly to our system. ${ }^{37}$ Our hierarchically nanostructured platform accelerates the drug model release due to increased desorption of the dye from the nanofibers' surface, and 
through an accelerated transport when subjected to NIR light stimulation, due to water expulsion from the hydrogel. Up to now, the combination of those two mechanisms was not reported by any other research group.

This composite structure allows for on-demand drug delivery as a result of the water expulsion from nanostructured hydrogel facilitating drug transport and the localized temperature rise, which increases the drug release rate. Moreover, our system avoids burst release phenomena resulting in a sustained release of drugs and avoiding a daily peak and trough drug levels. The drug can be delivered over a long period simultaneously with photothermal therapy for cancer or antibacterial treatments using the synergic action of multitherapy for more efficient cell killing. ${ }^{38}$ Such an approach can help overcome the insufficient drug release at target sites, which is the major limitation of conventional release systems.

\section{RESULTS AND DISCUSSION}

Hierarchical Levels of the Bioinspired Platform. The scheme reported in Figure 1a shows the hierarchical structure of the pillow: a nanostructured hydrogel with embedded gold nanorods (AuNRs) enclosed between two layers of nanofibrous material with encapsulated Rhodamine B (RhB). Several features of the developed bioinspired drug delivery system make it similar to the jellyfish's mesoglea (Figure 1b,c). First, by integrating the poly(L-lactide) (PLLA) nanofibrous structure with the hydrogel, we developed a layered and porous structure, which consists of randomly distributed fibers embedding a nanostructured hydrogel (Figure 1d). Second, our pillow system shows temperature-dependent swelling and anisotropic shrinking/swelling properties in aqueous solutions with different temperatures; i.e., the pillow swells in the axial direction at room temperature, but when the temperature increases, it also shrinks in the radial direction, causing a change in its shape from planar to bowl-shaped (Figure S1). Jellyfish mesoglea has a similar behavior, but to a different stimulus, i.e., $\mathrm{pH}^{34,37}$ However, several studies have also demonstrated temperature sensitivity properties of mesoglea. ${ }^{34}$

Each element of the pillow was deeply characterized to investigate its chemical composition, structure, and morphology, as well as to confirm that the developed system is suitable for its suggested final application.

Nanostructuration of Thermoresponsive Plasmonic Hydrogels Embedding Gold Nanorods. As shown in Figure 2a,b, AuNRs used in this study have a uniform size about $42 \pm$ $3.5 \mathrm{~nm}$ in length and $10 \pm 1.1 \mathrm{~nm}$ in width. The elemental color mapping and EDX spectra confirm the presence of pure gold nanorods in the precursor suspension (Figure $2 \mathrm{c}, \mathrm{d}$ ). As shown in Figure 2e, dynamic light scattering (DLS) results showed two peaks, one with an average hydrodynamic diameter of around 2 $\mathrm{nm}$ and a second one at $38 \mathrm{~nm}$. The most intense peak at $2 \mathrm{~nm}$ does not correspond to the actual particle size but is an artifact associated with the rotational diffusion of the nanorods. ${ }^{39}$ The peak at $38 \mathrm{~nm}$ is the hydrodynamic diameter of AuNRs, which confirms the results obtained by TEM analysis (Figure 2a). It is worth remembering that DLS commercial equipment is generally developed to analyze spherical objects; as a matter of fact, it can analyze only the equivalent hydrodynamic behavior, and therefore, it is difficult to distinguish between the short and long axes of nonspherical objects. Due to the surface plasmon resonance of AuNRs, light absorption peaks are found at 514 and $810 \mathrm{~nm}$, corresponding to the transversal and longitudinal absorbance bands of AuNRs, respectively (Figure 2f). The weak transverse band, which has a wavelength similar to the gold nanospheres, is insensitive to the nanorod aspect ratio (length/ width). The longitudinal band can be red-shifted when the nanorod aspect ratio is increased. ${ }^{40}$ For a AuNR with an aspect ratio of around 4, as in our case, the longitudinal band is close to the wavelength of the laser units typically used in photothermal therapy and well within the NIR biological window.

Among the thermoresponsive hydrogels that undergo reversible phase transitions at specific temperatures, PNIPAAm has been the one most frequently studied. ${ }^{41-43}$ The coil-toglobule transition of the polymer consists of at least two different thermal processes. ${ }^{18}$ First, water molecules around the hydrophobic isopropyl group and hydrophilic amide groups can rearrange to bulk water. Second, intra- and interchain hydrogen bonds are created between amide groups, and this phenomenon is accompanied by a loss in polymer chain-water hydrogen bonds. ${ }^{44}$ This transition depends on several factors, e.g., polymer concentration, the type and concentration of the cross-linking agent, and copolymerization with other monomers. In order to increase the low critical solution temperature (LCST), we copolymerized two monomers NIPAAm and NIPMAAm. The LCST of PNIPAAm in water is around $32{ }^{\circ} \mathrm{C}$, while the LCST value of PNIPMAAm is higher, around $42{ }^{\circ} \mathrm{C}$. According to the literature, ${ }^{45,46}$ the LCST of the resulting copolymer with a lesser quantity of NIPMAAm (as in our case) should increase to around $33-34{ }^{\circ} \mathrm{C}$ when macromolecule chains are not crosslinked. However, as confirmed by DSC results (Figure S2), the LCST of our hydrogel was around $37^{\circ} \mathrm{C}$. Moreover, it is worth noticing that the peak is broad and starts around $34^{\circ} \mathrm{C}$, while it ends at $42{ }^{\circ} \mathrm{C}$. This increase in LCST and broadening of the DSC peak are due to the presence of cross-linker molecules in the final polymer network. ${ }^{47}$ The use of $N, N^{\prime}$-methylene bisacrylamide (BIS-AAm) in our case shifted the LCST and led to the formation of a broad peak due to the faster reaction of BIS-AAm than NIPAAm and NIPMAAm during the polymerization process. ${ }^{48}$

High-resolution SEM was used to study the morphology of the hydrogel and reveal the possible presence of AuNRs on the surface of the PNIPAAm polymer (Figure $2 \mathrm{~g}$ ). Field emission SEM (FE-SEM) images of the P(NIPAAm-co-NIPMAAm)/ AuNR hydrogel were obtained by freeze-drying the prepared hydrogel. Higher magnification of the polymer surface showed no signs of protruding nanorods (Figure $2 \mathrm{~h}$ ). Moreover, TEM microscopy visualized the presence and distribution of AuNRs in the hydrogel, confirming the entrapment of AuNRs in the polymer matrix (Figure 2i). Elemental color mapping and EDX spectra confirmed the presence of pure AuNRs in the hydrogel (Figure $2 \mathrm{j}, \mathrm{k}$ ). Wide-angle X-ray scattering (WAXS) was used to compare the chemical structure of the developed plasmonic hydrogel with P(NIPAAm-co-NIPMAAm) control (Figure 21). Both sample diffractograms show their typical broad peaks at around 8.0 and $20.7^{\circ}$, which are attributed to the amorphous state of the polymer. The plasmonic hydrogel diffractogram shows two additional diffraction peaks at 38.0 and $44.2^{\circ}$, which correspond to AuNRs, while the signals related to the polymer network structure remain unchanged. ${ }^{49}$

Electrospinning of Nanofibers and Hierarchically Nanostructured Pillow Assembly. In our study, PLLA was selected as a material for electrospinning fibers, thanks to its excellent biological and mechanical properties and biodegradability. Moreover, this polymer shows significant drug release characteristics due to its high intermolecular forces binding dye/ drug molecules to the polymer surface in the presence of water. 

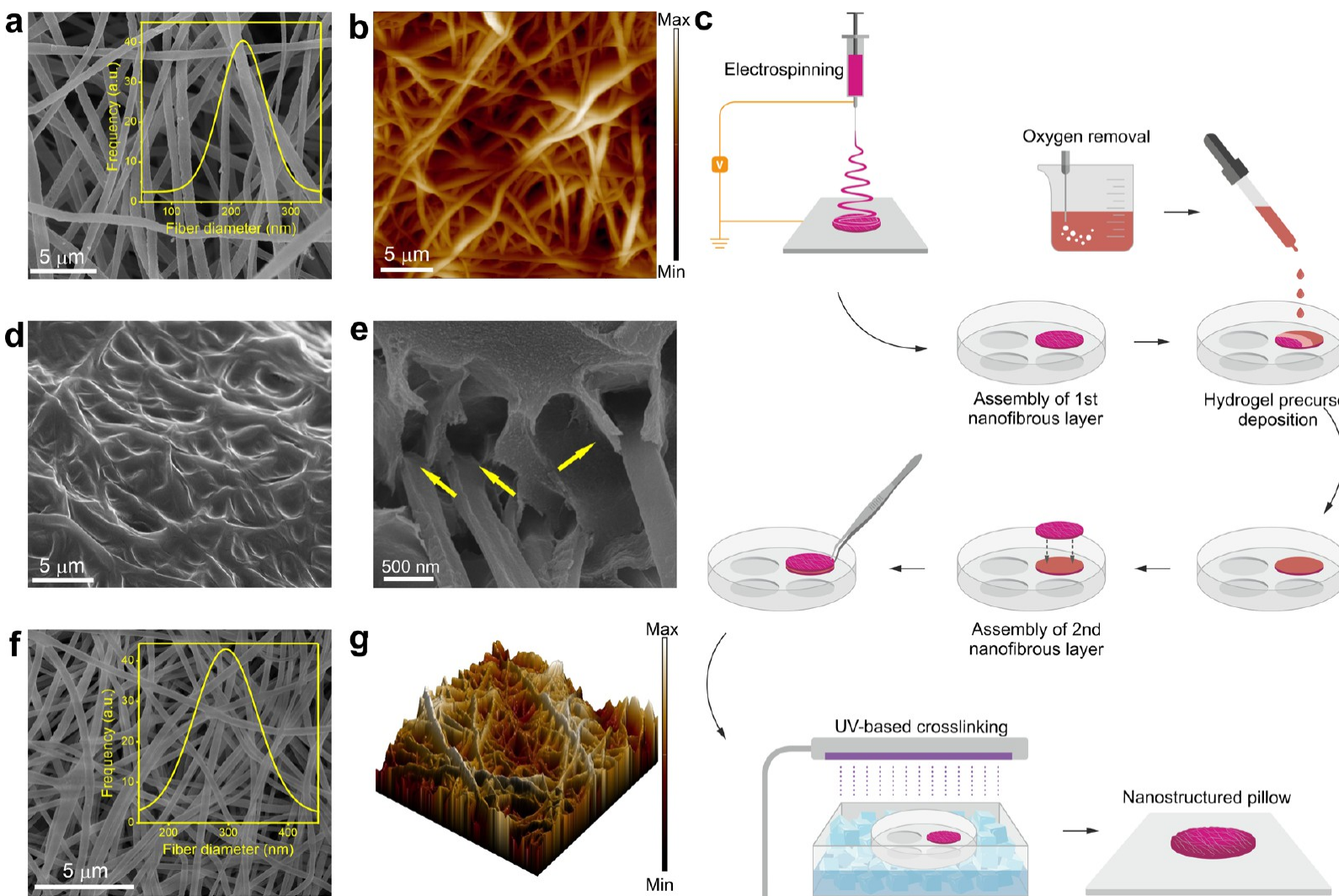

Figure 3. Morphological characterization of the electrospun nanofibers and nanostructured pillow fabrication. (a) SEM morphology of spun PLLA/ $\mathrm{RhB}$ nanofibers with a fiber diameter of $210 \pm 53 \mathrm{~nm}$. (b) The surface morphology of PLLA/RhB nanofibers observed with AFM shows an extremely rough nanofibrous material (the $z$-scale is equal to $5 \mu \mathrm{m}$ ). (c) Scheme showing the pillow preparation process starting from the electrospinning of PLLA/RhB nanofibers to the free-radical polymerization of the plasmonic hydrogel. (d) Top view of the PLLA/RhB nanofibers disassembled from the pillow showing the interpenetration of nanofiber pores with the hydrogel at the hydrogel-fiber interface. (e) Cross section of the pillow showing the nanofiber anchorage in the hydrogel mass (the yellow arrows indicate the anchoring points). (f) SEM morphology of PLLA/RhB nanofibers after pillow preparation and UV irradiation (the external side unconnected with the hydrogel) with the fiber diameter distribution showing an average fiber diameter of approximately $300 \pm 67 \mathrm{~nm}$. (g) Three-dimensional AFM image of the final pillow surface showing a typical porous nanostructure $(25 \mu \mathrm{m}$ $\times 25 \mu \mathrm{m} ; z$-scale: $5 \mu \mathrm{m})$.

According to the literature, most of the drugs released from nanofibrous PLLA materials do not exceed $20 \%$ of the cumulative release after several days or even weeks. ${ }^{28,29,50,51}$ One of the strategies that has been used quite often to increase the drug release rate from PLLA fibers is the addition of hydrophilic PEO, which may increase fiber porosity and promote drug desorption. ${ }^{52}$ However, such a strategy results in a burst release with shorter release times, while an approach that provides an externally triggered release could achieve a more suitable drug release mechanism.

First, we electrospun PLLA fibers loaded with $\mathrm{RhB}$ with a diameter of $210 \pm 53 \mathrm{~nm}$ (Figure 3a,b). The surface of the fibers is uniform and smooth, without beads along the fibers. The addition of $\mathrm{RhB}$ to the polymer solution resulted in a decrease in the average fiber diameter when compared to the pure PLLA fibers that measured around $862 \pm 307 \mathrm{~nm}$ in diameter (Figure S3). This decrease has been observed also in another study and is attributed to the increased conductivity of the polymer solution due to the presence of a cationic dye such as Rhodamine $\mathrm{B}^{28}$

A schematic representation of the pillow assembly process is shown in Figure $3 \mathrm{c}$. First, the nanofibrous nonwoven material loaded with the dye was electrospun on a drum collector. Two discs of $1.3 \mathrm{~cm}$ diameter were extracted from the mat, and one of them was placed into a Petri dish well, which served as a template. In the meantime, the hydrogel precursor solution was treated by bubbling argon gas for $10 \mathrm{~min}$ to remove any possible oxygen molecules, which might act as scavengers during the freeradical polymerization of the hydrogel. Then the precursor was gently dropped onto the nanofibrous material and covered with a second disc. Two nanofibrous layers were tightened at the edges, and finally, the nanostructured pillow was placed in an ice bath to maintain the material temperature below the LCST during the hydrogel polymerization and irradiated with UV light (power density of $225 \mathrm{~mW} / \mathrm{cm}^{2}$ ) for 5 min under controlled environmental conditions. The nanostructured pillow was characterized for its anchorage between the compartments of the system stability and also for photothermal and drug delivery capabilities.

After the preparation of several pillows, some of them were used to investigate the inner structure of the nanoplatforms developed. A few pillows were carefully delaminated, and the nanofibrous layers were removed to observe the internal part of the pillow and the contact of the fibers with the hydrogel. Figure $3 \mathrm{~d}$ shows the view of the nanofibrous surface that was in contact with the P(NIPAAm-co-NIPMAAm) hydrogel. We can clearly observe the interpenetration of the hydrogel within the nonwoven mat pores, which provides a physical hydrogelfiber interaction suitable for forming a very stable pillow structure. In addition, the view of the pillow cross section 

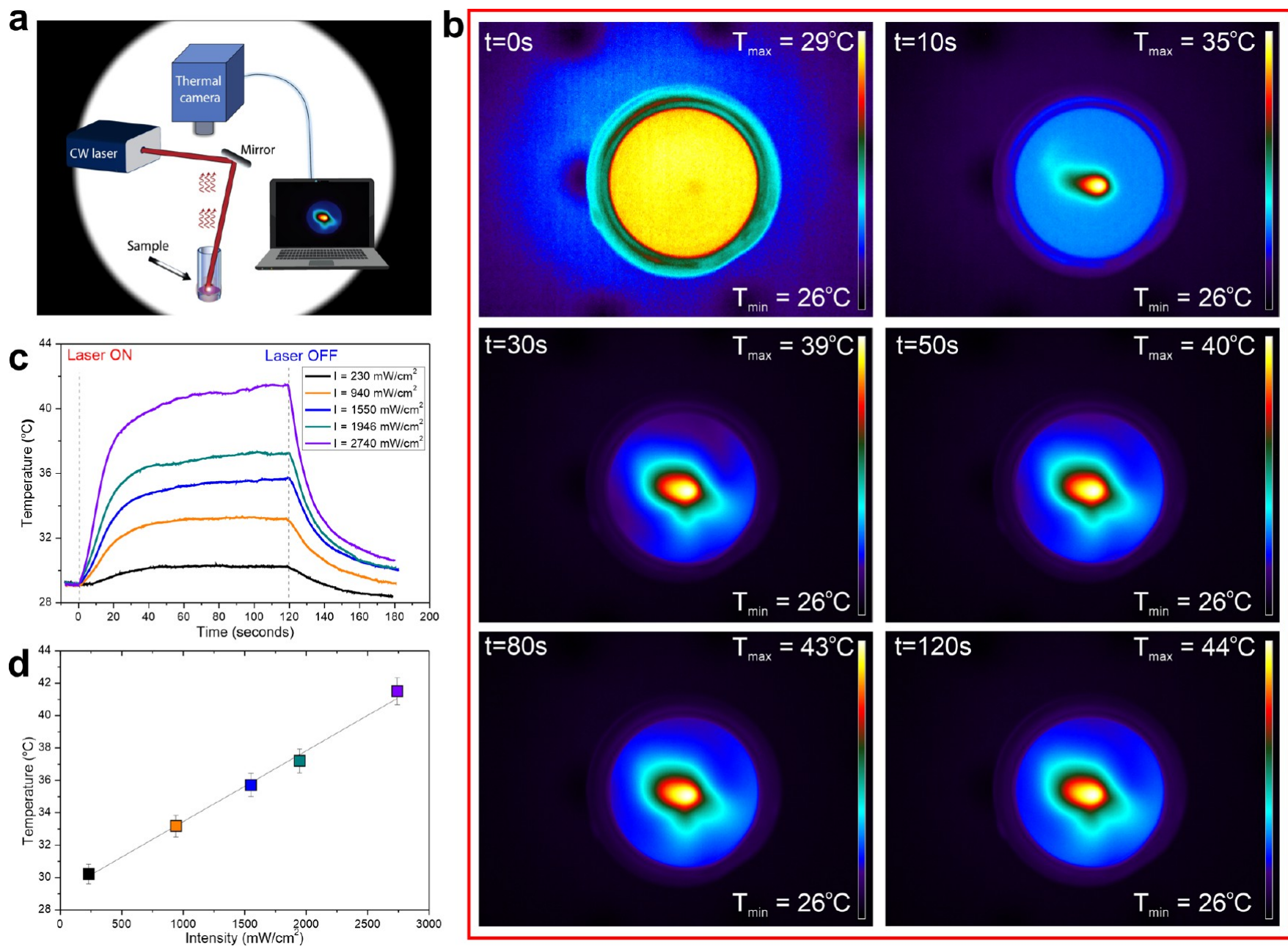

Figure 4. Photothermal properties of the nanostructured pillow showing the fast responsiveness of the developed plasmonic platform. (a) Scheme presenting an experimental setup where the laser beam runs from top to bottom, irradiating the pillow in a vial filled with water, which was observed with a thermal camera, while recording images directly on a computer. (b) Thermographic images captured at different times during the laser irradiation (intensity: $2740 \mathrm{~mW} / \mathrm{cm}^{2}$ ) of a floating single pillow. At time zero, water and the pillow show a similar level of temperature; at different time points, it is possible to visualize the typical temperature rise. (c) Temperature-time plots for various intensity values of the laser beam, temperature increases due to the capability of AuNRs to efficiently convert the incident light into heat energy. (d) Linear correlation between the maximum temperature detected and the intensity of the laser beam; colors correspond to the conditions described in panel (c).

obtained by FE-SEM shows the intimate anchorage of the first layer of fibers to the hydrogel mass (Figure 3e, anchorage sites are pointed with arrows). It is worth mentioning that the hydrogel does not penetrate the entire thickness of the nanofibrous mat but only a few micrometers.

The irradiation of the PLLA fibers with UV light during hydrogel cross-linking resulted in an increase in fiber diameter to around $300 \pm 67 \mathrm{~nm}$ (Figure 3f,g). The fiber swelling could be attributed to the increased distance between the molecular chains of the amorphous PLLA due to the interaction with UV light. Such a behavior of PLLA nanofibers was not observed in previous studies; ${ }^{53,54}$ however, the light power density used in our experiments was $10^{3}$ higher than in the abovementioned papers. As evidenced by gel permeation chromatography (GPC) analysis, there was no difference in the polymer molecular weight of the PLLA samples before and after UV irradiation (Figure S4, Table S1), thus proving the absence of any polymer degradation process triggered by the UV treatment. To assess the stability of the nanostructured pillow after laser irradiation, GPC (Figure S4, Table S1), SEM (Figure S5), XRD (Figure S6), FT-IR (Figure S7), and XPS (Figures S8, S9, Table S2) analyses were performed. None of these analyses showed any changes after 10 cycles of laser irradiation.

Fast Photothermal Responsiveness of the Plasmonic Nanoplatform. The NIR-stimulated thermoresponsive behavior of the plasmonic nanoplatform was investigated by irradiating the pillow with an NIR laser with an $810 \mathrm{~nm}$ wavelength in different irradiation intensities, up to a maximum power of $2740 \mathrm{~mW} / \mathrm{cm}^{2}$. It is well known that AuNRs show a strong absorption in the NIR region and that they can efficiently convert the absorbed energy to heat thanks to an excellent photothermal efficiency. ${ }^{55}$ The PLLA/P(NIPAAm-co-NIPMAAm) pillow embedding AuNRs inside the hydrogel structure shows a similarly strong absorption of the NIR laser and conversion to thermal energy due to the presence of the AuNRs. When the light is converted to heat and the temperature exceeds the hydrogel LCST, a cascade-like series of stimulus/response events lead to a rapid contraction of the hydrogel and water release due to the thermoresponsive properties of the P(NIPAAm-co-NIPMAAm) hydrogel. The experimental setup used to investigate the pillow's photothermal activity can be seen in Figure 4a, which shows a CW laser emitting a beam irradiating 

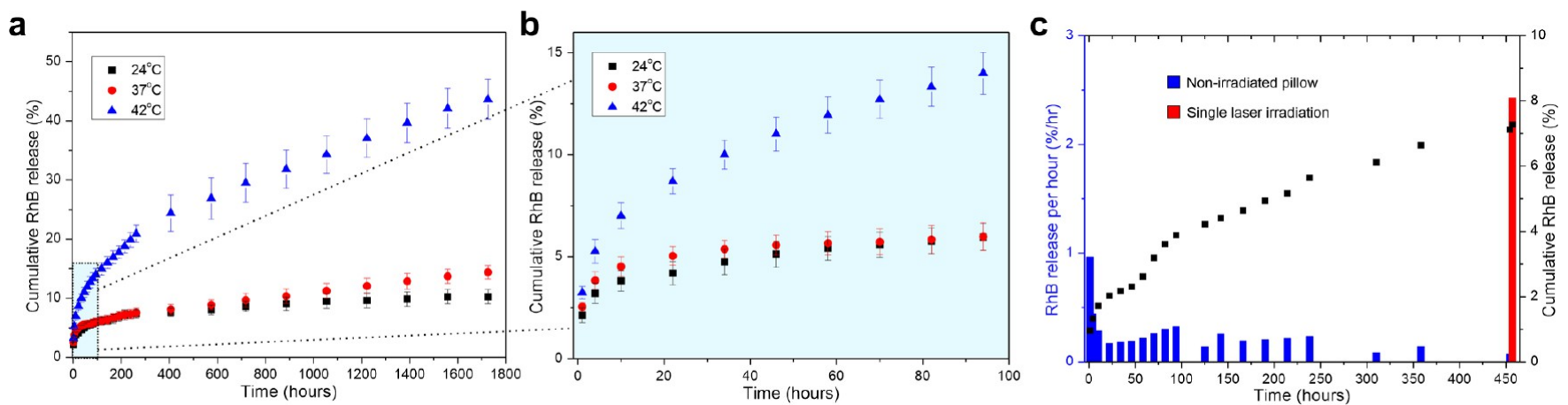

d
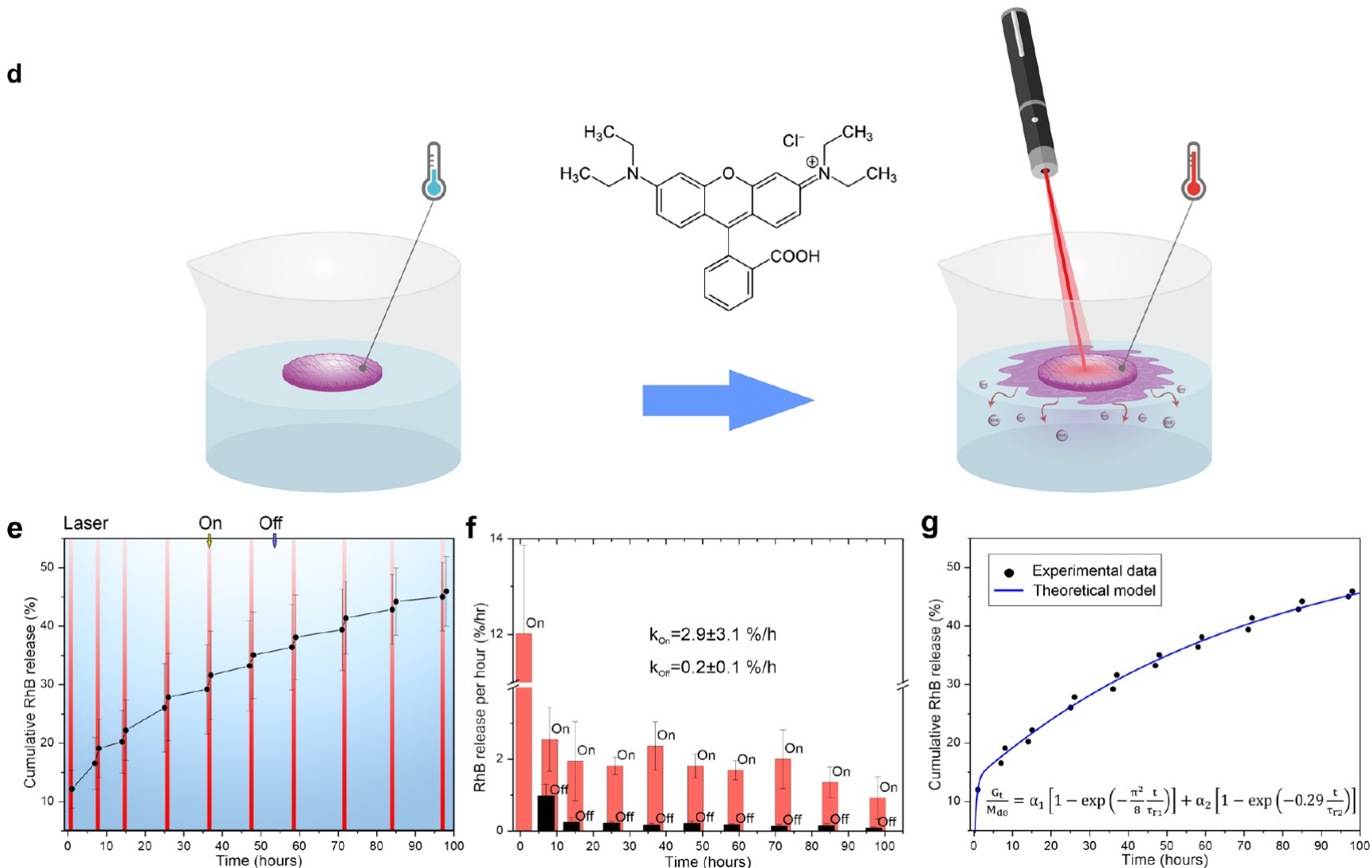

Figure 5. Drug delivery property of the nanostructured pillow. (a) Cumulative $\mathrm{RhB}$ release at three different temperature levels: 24,37 , and $42{ }^{\circ} \mathrm{C}$. (b) Cumulative release for the first $100 \mathrm{~h}$ as marked with light blue color in panel (a). (c) Cumulative release of RhB from a single pillow (black dots) presenting the influence of one-time laser irradiation on the final release, and corresponding columns presenting the release per hour, clearly showing the change in the dye released at different time stages and irradiation (compare the last blue column with the red column). (d) Scheme representing the temperature change that takes place during laser irradiation, which leads to a rise in the release of $\mathrm{RhB}$. (e) Cumulative $\mathrm{RhB}$ release after 10 laser irradiation cycles. The red vertical lines represent $1 \mathrm{~h}$ cycles of laser irradiation. (f) Column chart presenting the experimental RhB release per hour broken down into releases during laser irradiation (red - On) and without irradiation (black - Off). (g) Comparison between the proposed drug release theoretical model and experimental data. The black symbols correspond to the experimental data of RhB cumulative release after 10 laser irradiation cycles. The curve shows the results of mathematical model fitting considering both the first and second stages of drug release from eq 1 .

the pillow sample (contained in a water-filled vial) after passing through a custom-designed optical path. Perpendicular to the illuminated sample, a thermal camera records the thermographic images of the irradiated area and stores them on a computer. Figure $4 \mathrm{~b}$ shows the thermographic images with the temperature changes in the PLLA/P(NIPAAm-co-NIPMAAm)/AuNR pillow and the various irradiation times (intensity: $2740 \mathrm{~mW} /$ $\mathrm{cm}^{2}$ ). The temperature of the pillow increased significantly and rapidly, eventually leading to a slight rise in temperature of the surrounding water. The time-temperature profiles at different intensity values of the laser pump beam (from 230 to $2740 \mathrm{~mW}$ / $\mathrm{cm}^{2}$ ) are shown in Figure 4c. A significant and gradual temperature increase up to $42{ }^{\circ} \mathrm{C}$ upon irradiation can be seen (Figure $4 c$, Laser On) due to the capability of AuNRs to efficiently convert the incident light into heat energy. As soon as the pump beam is turned off (Figure 4c, Laser Off), the sample cools back down to room temperature (in less than $1 \mathrm{~min}$ ). Figure $4 \mathrm{~d}$ shows a linear correlation between the maximum temperature reached by the nanostructured pillow and the intensity of the pumping beam. This behavior can be justified by considering that the heat power density of AuNRs under laser beam irradiation is proportional to the square of the incident electric field, as described elsewhere. ${ }^{56}$ Certainly, the higher laser power could lead to a faster heating efficiency of the pillow 
sample. The maximum temperature of the pillow could be increased with increased gold nanorod concentration in the hydrogel and reach even $78{ }^{\circ} \mathrm{C}$ (Figures S10, S11). The plasmonic system, however, was designed to reach a temperature able to trigger hydrogel structural changes and, in turn, to control the drug release, as well as to reach the typical temperature range for performing PTT.

Near-Infrared Light-Triggered Cascade-like Responses Controlling Drug Delivery On Demand. The main goal of controlling drug delivery is to optimize its release rate in order to maintain the drug level in a therapeutic window by targeting specific tissues and cells while minimizing the drug side effects. With the cyclic On/Off pattern of laser irradiation, the pulsatile release of dyes/drugs can be modulated according to therapy requirements. One of the advantages of our system is the protection of the drug from thermal degradation, obtained by using cascade stimuli for controlling the drug release. Compared to a system where drugs are directly attached to the gold nanorods, nanofibers have the potential of releasing certain temperature-sensitive proteins, e.g., antibodies.

To emphasize the impact of laser irradiation on the dye release from the pillow, we first analyzed the release at different temperatures: 24,37 , and $42{ }^{\circ} \mathrm{C}$ (Figure 5a,b). A temperature of $24{ }^{\circ} \mathrm{C}$ was selected due to the fact that, when nonirradiated, the temperature of the pillow placed on the human hand is around room temperature. Additionally, it allows for the analysis of drug release in a scenario where the hydrogel is in a fully hydrated state (Figure S12, Supporting Information). A temperature of 37 ${ }^{\circ} \mathrm{C}$ was selected not only because it is the human body temperature but also because it is the LCST for our P(NIPAAmco-NIPMAAm) polymer: a temperature at which the hydrogel within the pillow starts to shrink and expels portions of water. A temperature of $42{ }^{\circ} \mathrm{C}$ was set based on the maximum temperature reached during photothermal responsiveness tests and because it is considered an ideal temperature level for effectively performing photothermal therapy. ${ }^{9}$ At $24{ }^{\circ} \mathrm{C}$, the hydrogel within the pillow is in its fully swollen form, and the drug release starts with about $2 \%$ release in the first hour (Figure $5 \mathrm{a}, \mathrm{b})$. Under $48 \mathrm{~h}$, the cumulative release at $24^{\circ} \mathrm{C}$ is lower than the release obtained at $37^{\circ} \mathrm{C}$. Finally, at $37^{\circ} \mathrm{C}$, the dye release is higher for much longer release times, around $400 \mathrm{~h}$. At $42{ }^{\circ} \mathrm{C}$, the release is reported to be 3 -fold higher than that registered at $37^{\circ} \mathrm{C}$. The difference in release curves is mainly based on the different dye desorption and diffusivity processes at various temperatures. To visualize the impact of the laser on the dye release, we show the cumulative release from a single pillow (Figure 5b), along with the corresponding dye release per hour of release (shown in columns). After $450 \mathrm{~h}$ of release experiment without laser irradiation at room temperature, we singly irradiated the pillow for $1 \mathrm{~h}$ with an $810 \mathrm{~nm}$ laser. The release increased significantly from around 0.1 (the last blue column in Figure $5 c$ ) to $2 \% / h$ (red column). This shows that even at longer experimental times, when the release rate is already moderate and the drug delivery system appears exhausted, the mutual effects of the hierarchically structured nanoplatform and its irradiation with NIR-light can enhance the release rate and finally trigger a therapeutic action, thus proving the possibility to extend the drug delivery system lifetime.

During the main drug model delivery tests, nanostructured pillows immersed in $1 \mathrm{~mL}$ of water were irradiated by a laser beam in a cyclic pattern, with one cycle lasting $1 \mathrm{~h}$ and for a total 10 cycles (Figure 5d). The Laser Off periods ranged from $6 \mathrm{~h}$ at the beginning to $12 \mathrm{~h}$ after several cycles. After the first cycle of laser irradiation (Figure 5e, vertical red line), the release was around $12 \%$, while during the Laser Off cycle, it took $6 \mathrm{~h}$; the release increased cumulatively up to $18 \%$ (Figure 5e, Figure S13, Supporting Information). The next irradiation cycle increased the release by more than $2 \%$, similar to additional laser irradiation cycles (Figure 5f, red columns). It can be seen that when data are presented as release per hour of the ongoing experiment, the stimulated release of drugs when the laser is On is much higher than that during the laser Off cycle $\left(k_{\text {on }}=2.9 \pm\right.$ $\left.3.1 \% / \mathrm{h} ; k_{\text {off }}=0.2 \pm 0.1 \% / \mathrm{h}\right)$. The trend of the released $\mathrm{RhB}$ per hour shows a decreased release rate with subsequent irradiation cycles. After $100 \mathrm{~h}$ of the release experiment, however, around $45 \%$ of $\mathrm{RhB}$ was already released compared to only $14 \%$ of $\mathrm{RhB}$ after releasing at $42{ }^{\circ} \mathrm{C}$ (compare Figure 5a (blue) and Figure $5 e)$. It is worth mentioning that after the Laser On cycle, when the pillow and the surrounding medium were heated by the laser to $42{ }^{\circ} \mathrm{C}$, the temperature of the release medium in the Laser Off cycles was maintained at $24{ }^{\circ} \mathrm{C}$ (Figure S12, Supporting Information).

According to the results reported in several articles, ${ }^{57-59}$ the mechanism of dye release from electrospun nanofibers is based on the desorption of the drug model from the nanopore surface and its further diffusion in water through the pores of the material. Diffusion of the dye in water takes place much faster than desorption, and therefore, desorption is the limiting factor in drug release from nanofibers. During the laser irradiation of the pillow, the kinetics of both desorption and diffusion rise as a result of the temperature increase. Due to the SPR effect, the generated heat not only does increase dye desorption and diffusive transport from the nanofibers, but an additional stage also takes place. During laser irradiation, the plasmonic hydrogel mass embedded between nanofibrous layers shrinks and the water expelled during the structure contraction flows through nanofibers (Figures S14, S15, Supporting Information). This can introduce a convective component that makes desorption much easier. The final equation considering the first and second stages of drug release from the pillow can be described by eq 1 .

$$
\frac{G_{t}}{M_{\mathrm{d} 0}}=\alpha_{1}\left[1-\exp \left(-\frac{\pi^{2}}{8} \frac{t}{\tau_{\mathrm{r} 1}}\right)\right]+\alpha_{2}\left[1-\exp \left(-0.29 \frac{t}{\tau_{\mathrm{r} 2}}\right)\right]
$$

$G_{t}$ is the amount of dye released by time $t$, the nanoporosity factor $\alpha_{1}=M_{\mathrm{sd} 0} /\left(M_{\mathrm{sd} 0}+M_{\mathrm{bd} 0}\right)<1$, with $M_{\mathrm{sd} 0}$ and $M_{\mathrm{bd} 0}$ being the initial amount of drug on the nanofiber surface (in particular, on the nanopore surface) and the initial amount of drug embedded in the fiber bulk, respectively; the total initial amount of drug in the fibers being $M_{\mathrm{d} 0}=M_{\mathrm{sd} 0}+M_{\mathrm{bd} 0} . \tau_{\mathrm{r} 1,2}$ are the characteristic time of the release process. The detailed explanation of the entire process, as well as the mathematical model that quantitatively describes the on-demand drug release mechanism, can be found in the Supporting Information. The theory described in previously published articles ${ }^{57,58}$ was amended by explaining the fact that, in the present case, the second stage is facilitating the dye release during laser irradiation. During this stage, the additional $\mathrm{RhB}$ release associated with the convective transport of the dye will proceed with the rate of the hydrogel's shrinking. After fitting the parameter values of eq 1 to the release profile, a perfect match between experimental data and theoretical model can observed (Figure $5 \mathrm{~g}$ ). The characteristic time for the drug release is within the order of magnitude of a typical duration of release processes, i.e., $90 \mathrm{~h}$ (Table S3). The characteristic time for the second stage during the laser 
a

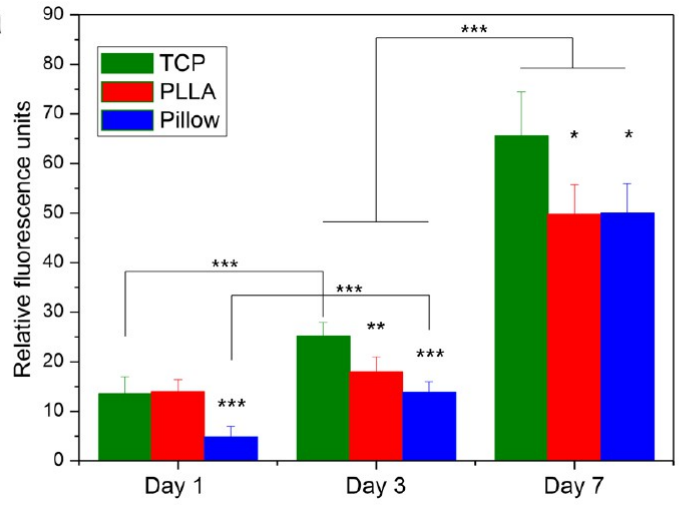

C
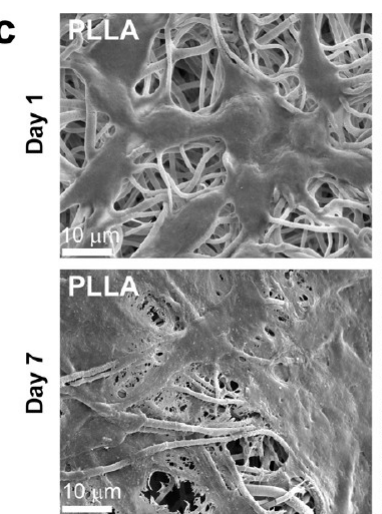

b
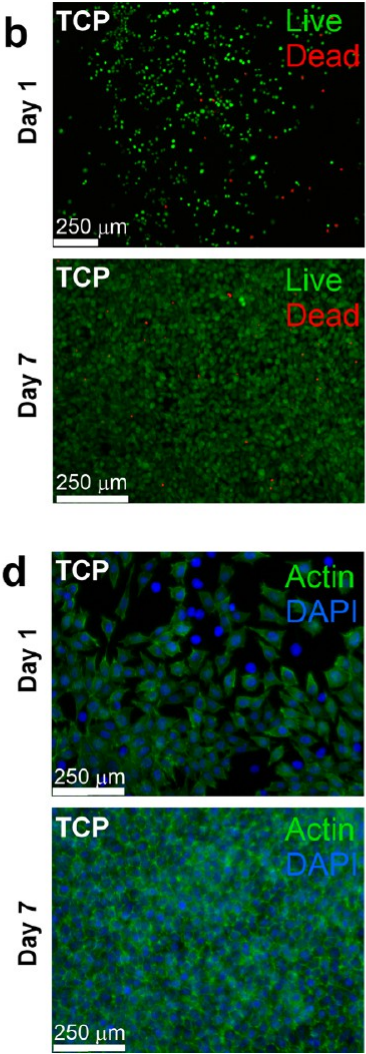
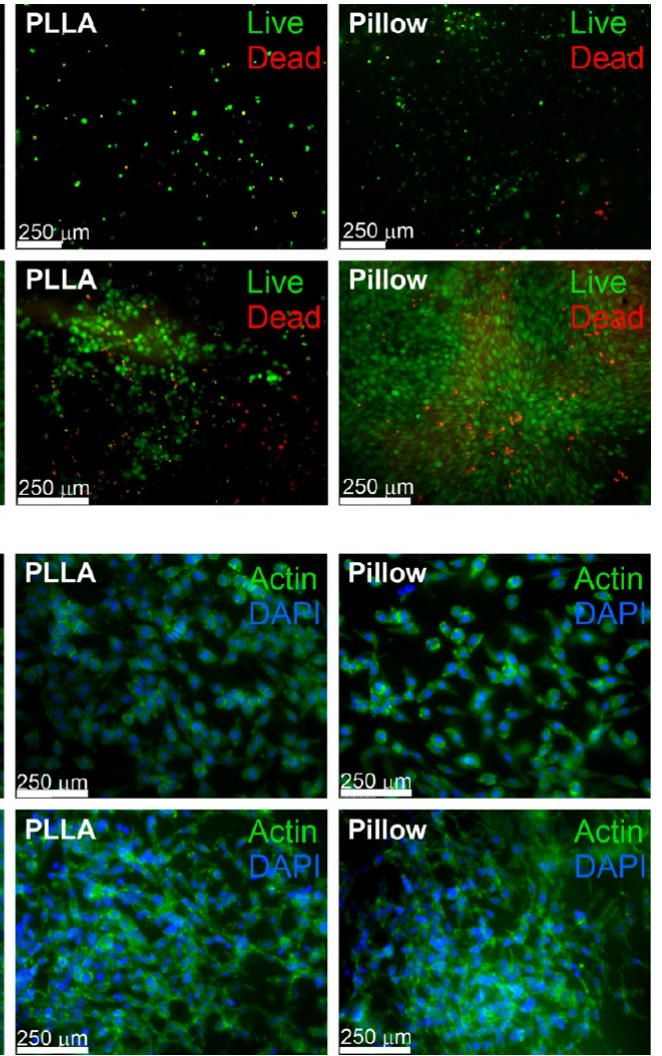

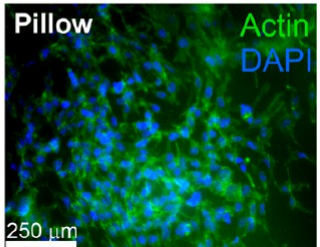

Figure 6. Biocompatibility of the pillow and evaluation of the cell-material interaction. (a) L929 cell line viability after 1, 3, and 7 days of culture, measured with PrestoBlue for TCP (control), electrospun PLLA nanofibers, and nanostructured pillows. (b) Live/Dead assay micrographs after 1 and 7 days of cell culture on TCP (control), electrospun PLLA nanofibers, and pillow: cells were stained with dyes, green fluorescent for alive cells and red fluorescent for dead ones. (c) SEM images presenting L929 cells on the surface of an electrospun PLLA mat and PLLA/P(NIPAAm-co-NIPMAAm) pillow after 1 and 7 days of culture, showing cell spreading and elongation as well as an increased number of cells during the culture time. (d) Fluorescence micrographs of L929 cells' nuclei (blue) and cytoskeletons (green), after 1 and 7 days of culture on TCP (control), electrospun PLLA nanofibers, and the nanostructured pillow. Significant differences: ${ }^{*} p<0.05, * * p<0.01$, and $* * * p<0.001$ are determined relative to TCP.

irradiation is much shorter $(0.15 \mathrm{~h})$ and within the order of magnitude of the shrinking of the hydrogel. In summary, the mathematical model well describes the process of drug desorption and its transport to the surrounding aqueous media, as shown in Figure 5g.

Cellular Response and Biocompatibility of the Biomimetic Pillow. L929 cells were cultured on the control sample (TCP), pure PLLA nanofibrous material, and on the pillow for 1, 3, and 7 days, while the cell viability was evaluated by the PrestoBlue and Live/Dead assays. In the PrestoBlue assay, the weakly fluorescent resazurin-based solution (purple), which acts as a cell viability indicator, is modified by the reduced environment of the viable cell and turns to a highly fluorescent pink resazurin, making possible the quantitative measure of the cell number over culture time. All materials were able to support the growth of L929 cells. It was seen that after 3 days of culture, the cell number increased significantly for TCP and the nanostructured pillow compared to day 1 (Figure 6a). For PLLA, the increase was not evident, and cultured cells on the fibrous microarchitecture presumably had a lower attachment rate. Greater cell numbers for all materials were seen from day 3 to day 7, while the values for the PLLA nanofibrous material and the PLLA/P(NIPAAm-co-NIPMAAm) pillow are notably different from those for TCP. This is due to the fact that cells cultured in $2 \mathrm{D}$ have an easier access to nutrients and migrate easily. The live and dead assay showed that after 7 days of culture, the number of dead cells on the pillow and PLLA nanofibers was higher than that for TCP (Figure $6 \mathrm{~b}$ and Figure S16). However, the viability of the cells for both materials is over $75 \%$, and considering the PrestoBlue results, both materials can be considered non-cytotoxic. Moreover, the in vitro cell response was also tested under NIR light irradiation to demonstrate its safety for biomedical application. Viability tests were performed after exposing cells to NIR light up to $15 \mathrm{~min} /$ day (Figures S17S19, Supporting Information). Results revealed that NIR irradiation did not negatively affect cell viability, comparable with nonirradiated samples (Figure 6a,b, Figure S10). Thus, NIR light exposure (as used in this study) can be considered safe for biomedical platforms.

To prove that the surface of nanofibrous materials can support the cell attachment and guarantee a suitable microenvironment for cell functioning, SEM and fluorescence microscopy were used to observe the morphology of cells cultured on PLLA and the PLLA/P(NIPAAm-co-NIPMAAm) pillow. The fibroblasts observed with SEM were well-spread, displaying a healthy spindle-like morphology, while the cell-matrix adhesion between L929 cells and PLLA/P(NIPAAm-co-NIPMAAm)/ AuNR pillows was also observed (Figure 6c). From day 1 of the culture, for both PLLA and the nanostructured pillow, the cell cytoskeleton stained with actin appeared spread and elongated with extended lamellipodia and filopodia (Figure 6d). The increased number of cells was also observed during the culture time, thus supporting the PrestoBlue assay results. These results suggest that the pillow offers a biocompatible surface supporting 
a

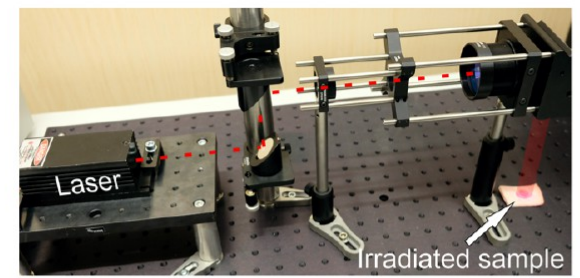

b
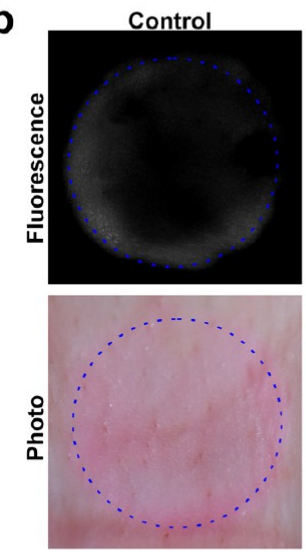

C

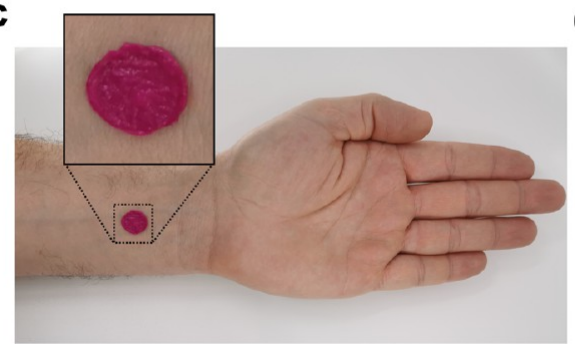

d

Control
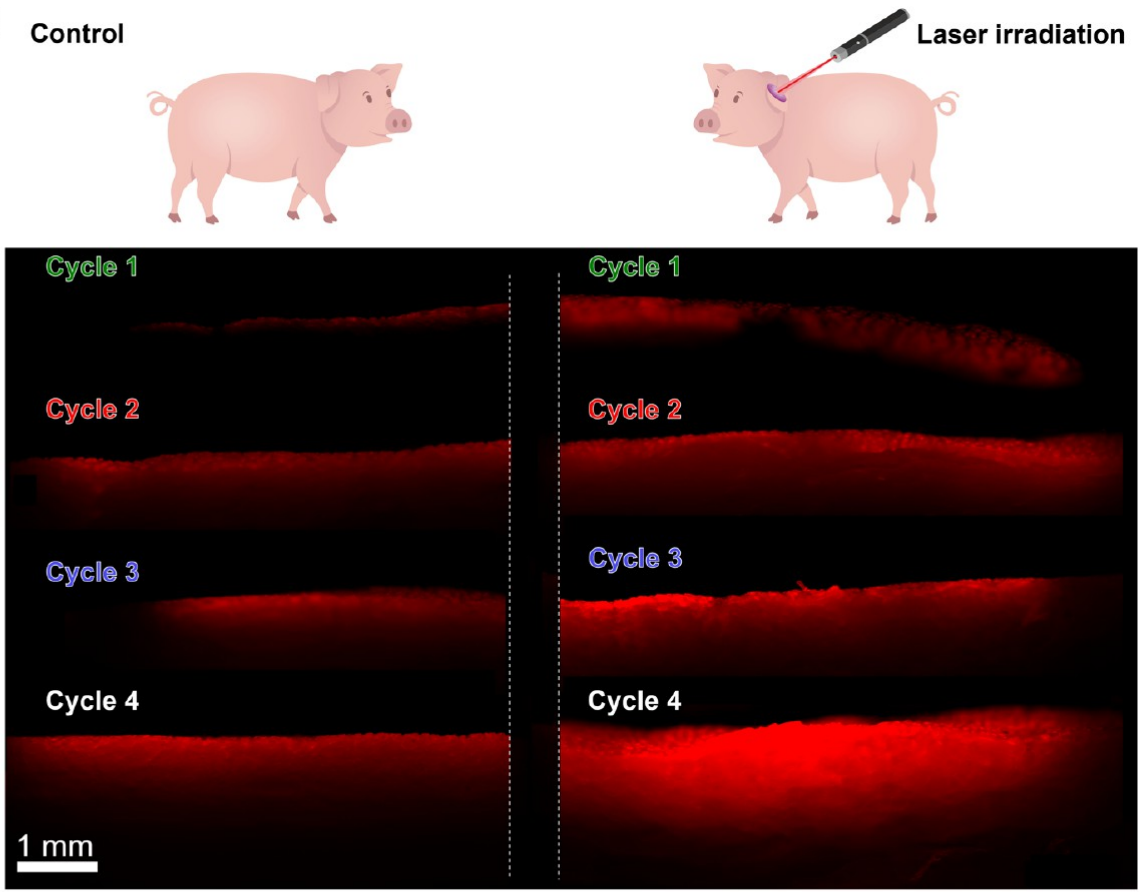

e

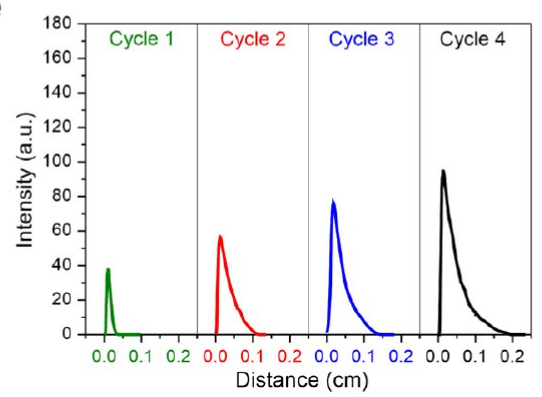

f

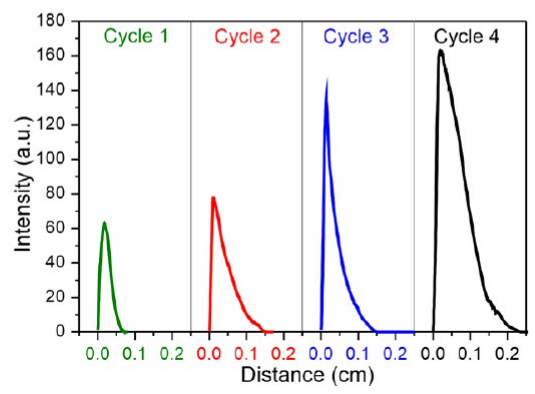

Figure 7. Ex vivo studies evaluating an externally regulated application of the drug model, releasing from the pillow and penetrating into a biological tissue. (a) Experimental setup showing the NIR laser source and the optical elements that make it possible to irradiate a nanostructured pillow positioned on a piece of pig skin. (b) Top-view fluorescence images and photographs of the pig skin for control experiments without NIR-laser irradiation and after one $15 \mathrm{~min}$ irradiation cycle. (c) Proposed use of a nanostructured pillow on the human skin tissue. (d) Pig skin cross section presenting $\mathrm{RhB}$ fluorescence and its transport across the skin for control experiments (no NIR-light irradiation) and NIR-laser irradiated samples in four 15 min cycles. (e) Fluorescence intensity chart presenting $\mathrm{RhB}$ penetration depth for control experiments (without NIR-laser irradiation) across the skin, showing a rise in fluorescence intensity with time. (f) Fluorescence intensity chart across the skin presenting the RhB penetration depth for four laser irradiation cycles, clearly showing a visibly increased penetration depth of RhB in the tissue depending on the number of cycles. Data reported in panels (e) and (f) were extracted when analyzing panel (b). Standard deviation for all the intensity curves in cycles was lower than $15 \%$.

cell viability, proliferation, and spreading. Therefore, it is suggested that with a laser-induced drug releasing function and good biocompatibility, the PLLA/P(NIPAAm-co-NIPMAAm) pillow may be used in polytherapy where on-demand enhanced drug bioavailability and phototherapy are needed.

Nanostructured Pillow Application for Enhancing Drug Penetration in Soft Tissues by NIR-Light Assistance. First, RhB internalization into L929 fibroblasts has been studied to prove the NIR light activation's impact on living matter. Fluorescence images reported in Figure S20 showed the $\mathrm{RhB}$ presence in the cell cultures. Consistently, control cells and cells in contact with pillows did not show any related fluorescence. On the other hand, samples loaded with $\mathrm{RhB}$ released the molecules during the culture time, revealing the presence of $\mathrm{RhB}$ in the culture. More specifically, $\mathrm{RhB}$ detection was the highest for samples subjected to NIR light irradiation, while nonirradiated samples showed lower RhB signals, according to the release rate. To evaluate the performance of the laser-triggered release of $\mathrm{RhB}$ in forming the nanostructured pillow, the release platform was applied to pig skin, which is commonly used as a surrogate for human skin. The transdermal drug delivery has generally many design challenges, but it provides several advantages as compared to, e.g., intravenous drug administration. ${ }^{60,61}$ These advantages include a more uniform pharmacokinetic profile of the drug in plasma and, most importantly, reduced off-target adverse effects. However, the outermost layer of the skin, the stratum corneum, constitutes a strong barrier for many molecules making it difficult for drugs to pass through the skin at clinically relevant rates. Drug-loaded electrospun fibers can provide sustained release and, if necessary, an additional on-demand dose of drug if subjected to NIR light stimulation. Fiber mats can be incorporated into wound dressings as part of a drug-releasing wound treatment technology. The nanostructured pillow was left on the continuously laser-irradiated skin for $15 \mathrm{~min}$ before examination (Figure 7a). The control experiment was performed under identical conditions, except that the laser was turned off. The fluorescence images under the microscope (Figure $7 \mathrm{~b}$ ) showed 
that the fluorescence of $\mathrm{RhB}$ delivered into the skin from the laser-irradiated pillow was much stronger than the one detected in the control. In the future, such platforms could be easily attached to the treated skin, as shown in Figure $7 \mathrm{c}$.

Histological images of the skin cross section after three additional cycles of laser irradiation were also taken to compare results further (Figure $7 \mathrm{~d}$ ). For each cycle, different skin fragments were used. The graphs in Figure 7e,f show averaged (from $2 \mathrm{~mm}$ distance) fluorescence intensity across the cut samples in order to visualize the drug model penetration depth. The fluorescence of $\mathrm{RhB}$ is hardly visible after the first cycle in the control experiment. The maximum intensity is approximately $40 \pm 3.9$ intensity units and increases according to the number of cycles up to $93 \pm 12$ intensity units at the fourth cycle. Most importantly, the penetration depth increases also. The distinct contrast between the fluorescent cross-sectional images (Figure $7 \mathrm{~d}$ ) provides additional evidence that more RhB was released into the skin from the laser-irradiated pillow, while $\mathrm{RhB}$ was delivered more deeply into the skin with laser-induced release. Altogether, these results demonstrate the feasibility of the proposed laser-induced drug delivery with simultaneous photothermal treatment.

\section{CONCLUSIONS}

In summary, we reported the successful fabrication of a drug delivery platform with biomimetic structural properties for the on-demand delivery of drugs. This platform consists of electrospun PLLA nanofibers loaded with a Rhodamine B drug model that encapsulates plasmonic hydrogel P(NIPAAmco-NIPMAAm)/AuNR. The paper describes the development of a simple strategy for significantly increasing drug delivery to a specific tissue. The release control is accomplished by cascadelike stimulation activated by NIR light, which is absorbed by AuNR and converted into heat, which, in turn, causes structural changes in the hydrogel and accelerates drug release kinetics. The pillow platform responsiveness was investigated using a series of analyses performed at various temperatures. The pillow showed a temperature-dependent drug delivery kinetics where the dye release is controlled by the increase in temperature. During laser stimulation cycles, we observed a 3-fold higher release of the dye than the temperature-controlled release, which can be regulated by implementing a certain number of On/Off cycles.

The light-activated multifunctionality of our system plays a vital role in the development of a platform for combined therapies (e.g., integrating the on-demand delivery of bioactive molecules and photothermal therapy). For instance, especially in the eradication of melanomas, PLLA/P(NIPAAm-coNIPMAAm)/AuNR pillows can be implanted into the postoperative bed after the removal of the tumor and additionally NIR light-treated to kill the remaining cancer cells. The same process can be applied to nonhealing, infected wounds where a laser-triggered pillow could release locally antimicrobials, while the generated heat would decrease bacterial colonies. ${ }^{62,63}$ Because such a platform successfully meets the desired requirements of biocompatibility and stability for thermoresponsive nanomaterials, the PLLA/P(NIPAAm-co-NIPMAAm)/AuNR system is an excellent candidate for achieving on-demand drug release in synergy with photothermal treatment.

\section{METHODS AND EXPERIMENTS}

Materials. Poly(L-lactide) (PLLA, PL18, Corbion Purac, Netherlands), chloroform ( $\mathrm{CHCl}_{3}$, POCh, Poland), $\mathrm{N}, \mathrm{N}$-dimethylformamide (DMF, POCh, Poland), $N$-isopropylacrylamide (NIPAAm, 97\%, Sigma Aldrich, Poland), $N, N^{\prime}$-methylene bisacrylamide (BIS-AAm, 99.5\%, Sigma Aldrich, Poland), N-isopropylmethacrylamide (NIPMAAm, 97\%, Sigma Aldrich, Poland), 2-hydroxy-4'-(2-hydroxyethoxy)-2methylpropiophenone (Irgacure 2959, 98\%, Sigma Aldrich, Poland), Rhodamine B (RhB, 95\%, Sigma Aldrich, Poland), gold nanorods (AuNRs, $\lambda_{1}=800 \mathrm{~nm}, \mathrm{OD}=50, c_{\mathrm{AuNR}} \approx 1 \mathrm{mg} / \mathrm{mL}$ nanoComposix, USA), fibroblasts L929 (Sigma-Aldrich, Poland), DMEM (ThermoFisher Scientific, USA), FBS (F9665, Sigma Aldrich, Poland), penicillin (Sigma Aldrich, Poland), streptomycin (Sigma Aldrich, Poland), cell viability assay (PrestoBlue, Invitrogen, USA), ethanol (POCh, Poland), hexamethyldisiloxane (HMDSO, Sigma Aldrich, Poland), paraformaldehyde (PFA, Sigma Aldrich, Poland), glutaraldehyde (GTA, Sigma Aldrich, Poland), Live/Dead Fixable Dead Cell Stains (ThermoFisher Scientific, USA), Triton X (Sigma Aldrich, Poland), ActinGreen, and NucBlue Reagent (Invitrogen, USA) were used as received.

Preparation of Electrospun Nanofibers. The polymer solution used for electrospinning was prepared by dissolving $5.75(\mathrm{w} / \mathrm{w} \%)$ (PLLA) polymer in a mixture of $\mathrm{CHCl}_{3}$ and DMF 9:1 (w/w). Afterward, $\mathrm{RhB}$ was mixed with the polymer solution (1 wt \% with respect to the polymer). Rhodamine $\mathrm{B}$ was used as a model drug, soluble in a polymer solvent as well as in aqueous media. The PLLA nanofibrous material was electrospun using a $17 \mathrm{kV}$ positive voltage and with the flow rate set at $800 \mu \mathrm{L} / \mathrm{h}$. Fibers were collected on a grounded rotating drum collector at $500 \mathrm{rpm}$ and placed $15 \mathrm{~cm}$ away from a $26 \mathrm{G}$ needle tip. The temperature during electrospinning was around $23^{\circ} \mathrm{C}$, and humidity was 50-60\%. A PLLA nanofibrous material without Rhodamine B was prepared with the same parameters.

Preparation of the Hydrogel Precursor Solution with Dispersed Gold Nanorods. The hydrogel precursor solution (4.8 wt \%) was a mixture of NIPAAm, NIPMAAm, and BIS-AAm in a proportion of 1:0.05:0.03, dissolved in deionized water (95.2 wt \%). Irgacure 2959 was used at a concentration of $1 \mathrm{mg} / \mathrm{mL}$ as the photoinitiator of the polymerization reaction and was added to obtain a final concentration of $0.1 \mathrm{wt} \%$. Finally, a gold nanorod suspension was added to the hydrogel precursor solution in a proportion of 1:5, resulting in a $0.02 \mathrm{wt} \%$ AuNR concentration in the hydrogel precursor.

Nanostructured Pillow Fabrication. The pillow consists of two components: the nanofibrous material and the plasmonic hydrogel embedding gold nanorods. The fabrication steps for obtaining the pillow are reported in Figure 3c and Figure S21. For the preparation of a single nanostructured pillow, two discs of PLLA fibrous material were used (with an average weight of around $2.5 \mathrm{mg}$, diameter of $1.3 \mathrm{~cm}$, and thickness of around $100 \mathrm{um}$ ). A nanofibrous disc was placed in a 4-well Petri dish with a single well size $10 \mathrm{~mm}$ in diameter and $1 \mathrm{~mm}$ in depth (cat. No. 627170, Greiner Bio-One). A nanofibrous disc was carefully fitted into the well, and its edge was directed upward. Then, $50 \mu \mathrm{L}$ of the hydrogel precursor solution with AuNRs (after $10 \mathrm{~min}$ of oxygen removal by bubbling the solution with argon) was supplied and evenly spread onto the nanofibrous disc surface. Finally, a second electrospun disc was applied on the top to form a sandwich-like composite, while the edges of both discs were pressed with tweezers to ensure connection and tightness. The pillow was left in the Petri dish for $1 \mathrm{~min}$ before UV irradiation to ensure that the hydrogel precursor penetrated the pores of the nanofibrous material and permitted a proper anchorage of the pillow at the hydrogel-fiber interface. The prepared composite was irradiated under a UV lamp (Dymax, $400 \mathrm{~W}$, power density of $225 \mathrm{~mW}$ / $\mathrm{cm}^{2}$ ) for $5 \mathrm{~min}$ with the use of an ice bath in order to keep the temperature below $15^{\circ} \mathrm{C}$. After polymerization, the pillow was washed with deionized water.

Morphological Characterization. In order to study the AuNR shape, dimension, composition, presence, and distribution in the hydrogel matrix, transmission electron microscopy (TEM) was performed. For the analysis of the pure AuNR, the solution was dripped onto a TEM grid coated with a polymer film. In the case of the nanostructured hydrogel, freeze-dried samples were cut into small 
pieces and placed on a TEM grid. After $4 \mathrm{~h}$ of drying in a vacuum drier, samples were analyzed on an FEI Talos F200X TEM (applied voltage of $200 \mathrm{kV}$ ). Additionally, energy-dispersive X-ray spectroscopy (EDX) was performed in STEM mode. For this purpose, a high-angle annular dark field detector and a Super-X EDX system with four silicon drift detectors (SDDs) were used. Scanning electron microscopy (SEM) and field-emission SEM (FE-SEM) observations were performed using JEOL JSM-6390LV and FEI Nova NanoSEM 450 microscopes, respectively. Before the SEM and FE-SEM imaging, materials were sputtered with layers of gold approximately $5 \mathrm{~nm}$ thick using a SC7620 Polaron mini sputter coater (Quorum Technologies Ltd., Ashford, UK). The surface topography of the samples was evaluated using an atomic force microscope (AFM, Ntegra, NT-MDT) equipped with a silicon cantilever (NSG01, NT-MDT). The spring constant and mechanical resonance were in the range of $0.003-0.13 \mathrm{~N} / \mathrm{m}$ and $4-17$ $\mathrm{kHz}$, respectively. Measurements were performed in a semicontact mode, with a resonance frequency of $150 \mathrm{kHz}$ and $500 \times 500$ points per image.

Chemical Characterization. Ultraviolet-visible (UV-vis) spectra were acquired using a Multiskan GO spectrophotometer (Thermo Scientific, USA) to scan over a range of 400 to $1000 \mathrm{~nm}$ every $5 \mathrm{~nm}$. A dynamic light scattering (DLS) Zetasizer Nano ZS (Malvern, UK) was used to analyze the hydrodynamic diameter of AuNRs. In order to prove the presence of gold nanoparticles in the hydrogel matrix and detect any potential changes in the hydrogel polymer structure caused by the inclusion of nanorods or laser irradiation, wide-angle X-ray scattering (WAXS) analyses were performed. Measurements were done on a Bruker D8 Discover diffractometer in reflection mode, using the Bragg-Brentano geometry. The analysis was conducted in the angular range $(2 \theta)$ of $5-50^{\circ}$. Data were collected with a step of $0.02^{\circ}$ per $1.0 \mathrm{~s}$ in each point. The molecular mass and polydispersity of the polymers were determined in $\mathrm{CHCl}_{3}$ at room temperature by gel permeation chromatography (GPC) on an HPLC Lab Flow 2000 apparatus equipped with a Rheodyne 7725i injector, a Phenomenex Phenogel mixed-bed $5 \mu \mathrm{MXL}$ type column, and an RI K-2301 KNAUER detector. The eluent flux was $1 \mathrm{~mL} / \mathrm{min}$, and the calibration curve was obtained using monodisperse polystyrene standards.

Photothermal Property Evaluation. The thermo-optical setup uses a CW diode laser (Coherent Powerline) operating at $810 \mathrm{~nm}$ in the high absorption range of AuNRs (longitudinal plasmon band). The laser beam has a rectangular profile, but it was converted into an almost circular spot by means of a $20 \mathrm{~cm}$ focal length elliptical lens. A highresolution thermal camera (FLIR, A655sc) was used for mapping and identifying both the heating spatial distribution and the temperature profile under top-pumping laser illumination. The camera produces thermal images of $640 \times 480$ pixels with an accuracy of $\pm 0.2^{\circ} \mathrm{C}$. It works seamlessly with proprietary software (FLIR ResearchIR Max) to record and process the thermal data acquired by the camera.

Release from the Pillow at Different Temperatures. The release of Rhodamine $\mathrm{B}$ from the pillow was analyzed at three different temperatures: 24,37 , and $42{ }^{\circ} \mathrm{C}$. The release at $42{ }^{\circ} \mathrm{C}$ simulated the conditions during the laser irradiation obtained by the light-plasmonic material interaction. After preparation, each pillow was separately immersed in $1 \mathrm{~mL}$ of deionized water at the abovementioned temperatures. The vial was sealed and wrapped with aluminum foil to prevent water evaporation and light exposure. The supernatant fluid was exchanged with fresh water, maintained at the same temperature as set for any specific test, after $1,4,10$, and $12 \mathrm{~h}$ of release. The sample temperature was monitored by a thermocouple (accuracy $\pm 0.1{ }^{\circ} \mathrm{C}$ ) several times for each sample. Over the next 4 days, the release level of $\mathrm{RhB}$ was measured every $12 \mathrm{~h}$. Subsequently, the measurement time points were reduced to once per day (over the next 7 days) and then, finally, to one measurement per week. Fluorescence signals were measured with a precisely calibrated fluorometer (Fluoroskan Ascent TM Microplate Fluorometer, Thermo Scientific, USA) (excitation/ emission $485 / 538 \mathrm{~nm}$ ) and used to evaluate the concentration of released $\mathrm{RhB}$ based on a calibration curve.

Release from the Pillow under NIR Laser Irradiation. The experiments with pillow irradiation were carried out using an $810 \mathrm{~nm}$ $\mathrm{CW}$ diode laser (Coherent Powerline) and power density of $2740 \mathrm{~mW} /$ $\mathrm{cm}^{2}$, similar to that described Photothermal Property Evaluation. The pillow was suspended in a closed vial filled with $1 \mathrm{~mL}$ of deionized water. The vial was additionally secured with a parafilm seal to protect the sample from water evaporation. The pillow was laser-irradiated for 1 h. Afterward, the supernatant was collected and its concentration was measured using the fluorometer to evaluate the amount of $\mathrm{RhB}$ released. The pillow was then immersed in fresh deionized water (1 $\mathrm{mL}$ ), parafilm-sealed, and left at room temperature for $6 \mathrm{~h}$. Again, the supernatant concentration was measured, and the vial was refilled with fresh deionized water, parafilm-sealed, and laser-irradiated further. This procedure was repeated for 4 days with different release intervals at room temperature.

In Vitro Cell Study. In order to determine the cellular response to the prepared PLLA/P(NIPAAm-co-NIPMAAm) pillow, different studies were performed in direct contact with the materials. Cell viability tests, adhesion and spreading tests on the material surfaces, and nuclear and cytoskeleton stainings were performed with and without NIR light irradiation (Figures S17-S19, Supporting Information).

Cell Culture. Material cytotoxicity tests were performed using L929 fibroblasts. Cells were cultured in $75 \mathrm{~cm}^{2}$ flasks in a culture medium consisting of $89 \%$ Dulbecco's minimum essential medium (DMEM), $10 \% \mathrm{FBS}$, and $1 \%$ antibiotic (penicillin/streptomycin) and held in an incubator at $37{ }^{\circ} \mathrm{C}$ with $5 \% \mathrm{CO}_{2}$. The culture medium was changed every 3 days. For seeding, cells were harvested by washing them with PBS, adding $5 \mathrm{~mL}$ of $0.05 \%$ (0.25\%, diluted 5-fold with PBS) trypsin solution to the flask, and placed for $5 \mathrm{~min}$ in an incubator. Afterward, a gentle tapping on the side of the flask lifted the cells up from the bottom of the flask. The harvested cells, with the addition of $5 \mathrm{~mL}$ of culture medium, were then centrifuged for $5 \mathrm{~min}$ at $100 \mathrm{~g}$. The collected pellet was resuspended with the culture medium and diluted to the desired cell density.

Material Preparation and Cell Seeding. Samples were sterilized with UV light for $30 \mathrm{~min}$ on each side before cell culture. Samples were placed in a 48-well plate, and a suspension of L929 cells was placed on top of the materials. Cells were seeded with an initial density of 15,000 units $/ \mathrm{cm}^{2}$.

Cell Viability Test and Live/Dead Assay. After 1, 3, and 7 days of cell culture, the cell viability test was performed via a PrestoBlue assay, using a solution made of 9:1 culture medium/PrestoBlue reagent. The modified culture medium was added to each sample, according to the protocol, and samples were incubated for $1 \mathrm{~h}$. Then $150 \mu \mathrm{L}$ from each well was transferred to a 96-well plate, and data were collected using a fluorometer (Fluoroskan Ascent TM Microplate Fluorometer, Thermo Scientific, USA), with excitation at $530 \mathrm{~nm}$ and emission at $620 \mathrm{~nm}$. The Cell Live/Dead assay was performed with Live/Dead Fixable Dead Cell Stains. First, samples were transferred to new multiwell plates and washed with PBS three times. The dye solution ( $1 \mathrm{~mL} \mathrm{PBS,} 0.5 \mu \mathrm{L}$ calcein, $2 \mu \mathrm{L}$ ethidium homodimer) was added to samples, and subsequently, the latter was incubated for $10 \mathrm{~min}$. Finally, the dye solution was removed, and the samples were washed again with PBS. Observations were performed using a fluorescence microscope (Leica, AM TIRF MC, Germany).

Cell Morphology and Spreading. For characterizing cellular morphology and spreading on materials, SEM imaging and fluorescence staining were conducted. For SEM observations, cells were fixed with $2.5 \%$ GTA for $30 \mathrm{~min}$ and washed with PBS to remove any residual GTA. Then samples were dehydrated in a series of ethanol solutions (for $20 \mathrm{~min}$ each) at increasing concentrations (60, 80, and 100\%) followed by ethanol/HDMS solutions (2:1 and 1:2) and, as a final step, in a solution of $100 \%$ HDMS, in which they were left to dry under the fume hood. Finally, samples were coated with a gold layer. SEM observations were performed using a JEOL JSM-6390LV microscope.

For fluorescence microscopy observations, samples were fixed with a $3 \%$ PFA solution for $30 \mathrm{~min}$. In order to increase the permeability of the cell membrane for dyes, samples were incubated in Triton X-100 solution $(1 \% \mathrm{v} / \mathrm{v})$ for $5 \mathrm{~min}$. Then samples were immersed in an ActinGreen and NucBlue Reagent solution ( 2 drops $/ \mathrm{mL}$ in PBS) and incubated for $30 \mathrm{~min}$. Finally, the solution was replaced with PBS. The samples were observed using a fluorescent microscope (Leica, AM TIRF MC, Germany). 
Ex Vivo Test. For the analysis of the drug model release from the pillow and penetration through mammalian tissues, we selected pig skin as a model for dye transport evaluation after laser irradiation. The selection of such a model is also in agreement with the $3 R$ (Replacement, Reduction, Refinement) guiding principles for a more ethical use of animal testing. The pig's ears were obtained from a slaughterhouse, which was exempt from approval by the Local Ethical Committee for Experiments on Animals. The pig's ears were washed, shaved, and cut to pieces of $5 \mathrm{~cm}^{2}$ using a scalpel (Figure S22a). The pillow material was placed on the skin, covered with a hydrogel patch (Figure S22b), and irradiated with an $810 \mathrm{~nm}$ laser (power density 2740 $\mathrm{mW} / \mathrm{cm}^{2}$ ), for 15 min per cycle, for a total of four cycles. After each cycle, a slice of the ear was excised with a scalpel from the center of a circle that was marked by $\mathrm{RhB}$ during the contact with the pillow (Figure S22c,d). For each cycle, different pieces of pig skin were used. Finally, the slices were imaged using a fluorescence microscope (Leica, AM TIRF MC, Germany) in order to visualize the longitudinal cross section of the sample and quantify the drug model penetration into the tissue. A control experiment was performed under identical conditions but without laser irradiation at room temperature.

Statistical Analysis. The data obtained for cellular viability were expressed as mean \pm standard deviation (SD) and compared using a one-way ANOVA test and analysis of variance (Tukey's test). Data were considered as significantly different when $p<0.05$. Rhodamine B release experiments from pillows were performed in quintuplicate for each temperature and for laser-assisted experiments.

\section{ASSOCIATED CONTENT}

\section{SI Supporting Information}

The Supporting Information is available free of charge at https://pubs.acs.org/doi/10.1021/acsami.0c13266.

Video demonstrating the hydrogels at different temperatures (MP4)

Nanostructured pillow shrinking; DSC curves of P(NIPAAm-co-NIPMAAm); SEM images of PLLA nanofibers; GPC trace of PLLA after UV and $810 \mathrm{~nm}$ laser irradiation; FE-SEM micrographs of pillow after laser irradiation; WAXS diffractograms of PLLA from the pillow; Fourier transform infrared spectroscopy (FT-IR) analysis; X-ray photoelectron spectroscopy (XPS) analysis; photothermal responsiveness of the plasmonic nanoplatform for different AuNR concentrations; the pillow temperature during the application on the human hand; a mathematical model describing desorptionlimited drug delivery enhanced by water expulsion from the P(NIPAAm-co-NIPMAAm) hydrogel; Rhodamine B delivery from the nanostructured pillow with the curve from a mathematical model; hydrogel sphere during shrinking in water; shrinking dynamics of the P(NIPAAm-co-NIPMAAm) sphere; percentage of viable cells extracted from Live/Dead images; in vitro studies during NIR light irradiation; in vitro studies of Rhodamine $\mathrm{B}$ internalization; nanostructured pillow preparation process; ex vivo tissue preparation process (PDF)

\section{AUTHOR INFORMATION}

\section{Corresponding Author}

Filippo Pierini - Department of Biosystems and Soft Matter, Institute of Fundamental Technological Research, Polish Academy of Sciences, Warsaw 02-106, Poland; 이이. ord. 0000-0002-6526-4141; Email: fpierini@ippt.pan.pl

\section{Authors}

Pawel Nakielski - Department of Biosystems and Soft Matter, Institute of Fundamental Technological Research, Polish Academy of Sciences, Warsaw 02-106, Poland

Sylwia Pawłowska - Department of Biosystems and Soft Matter, Institute of Fundamental Technological Research, Polish Academy of Sciences, Warsaw 02-106, Poland

Chiara Rinoldi - Department of Biosystems and Soft Matter, Institute of Fundamental Technological Research, Polish Academy of Sciences, Warsaw 02-106, Poland

Yasamin Ziai - Department of Biosystems and Soft Matter, Institute of Fundamental Technological Research, Polish Academy of Sciences, Warsaw 02-106, Poland

Luciano De Sio - Research Center for Biophotonics and Department of Medico-Surgical Sciences and Biotechnologies, Sapienza University of Rome, Latina 04100, Italy; (1) orcid.org/0000-0002-2183-6910

Olga Urbanek - Laboratory of Polymers and Biomaterials, Institute of Fundamental Technological Research, Polish Academy of Sciences, Warsaw 02-106, Poland

Krzysztof Zembrzycki - Department of Biosystems and Soft Matter, Institute of Fundamental Technological Research, Polish Academy of Sciences, Warsaw 02-106, Poland

Michał Pruchniewski - Department of Biosystems and Soft Matter, Institute of Fundamental Technological Research, Polish Academy of Sciences, Warsaw 02-106, Poland Massimiliano Lanzi - Department of Industrial Chemistry "Toso Montanari", Alma Mater Studiorum - University of Bologna, Bologna 40136, Italy; 이이.org/0000-00022466-2813

Elisabetta Salatelli - Department of Industrial Chemistry "Toso Montanari", Alma Mater Studiorum - University of Bologna, Bologna 40136, Italy

Antonella Calogero - Research Center for Biophotonics and Department of Medico-Surgical Sciences and Biotechnologies, Sapienza University of Rome, Latina 04100, Italy

Tomasz A. Kowalewski - Department of Biosystems and Soft Matter, Institute of Fundamental Technological Research, Polish Academy of Sciences, Warsaw 02-106, Poland

Alexander L. Yarin - Department of Mechanical and Industrial Engineering, University of Illinois at Chicago, Chicago, Illinois 60607-7022, United States; 이이이.org/0000-0001-80322525

Complete contact information is available at: https://pubs.acs.org/10.1021/acsami.0c13266

\section{Author Contributions}

${ }^{\#}$ P.N. and S.P. contributed equally to this work. F.P. and P.N. conceived the idea and designed the experiments. S.P., C.R., and Y.Z. fabricated the nanoplatforms. P.N., S.P., C.R., Y.Z., O.U., and F.P. performed the pillow structural characterization. L.D.S. and A.C. designed and conducted the photoresponsiveness experiments. S.P., Y.Z., K.Z., and M.P. performed the drug delivery tests. O.U. and C.R. conducted cell studies. P.N., C.R., Y.Z., K.Z., and S.P. performed the ex vivo tests. M.L., E.S., and F.P. studied the nanomaterial stability. A.L.Y. developed the theoretical model. A.L.Y., P.N., and T.A.K. performed simulations. P.N. wrote the manuscript. All authors discussed the results and commented on the manuscript. F.P. supervised the project.

\section{Notes}

The authors declare no competing financial interest. 


\section{ACKNOWLEDGMENTS}

This work was supported by the National Science Centre grant no. 2015/19/D/ST8/03196, NAWA grant no. PPI/APM/ 2018/1/00045/U/001, and NAWA Canaletto grant no. PPN/ BIL/2018/2/00035/U/00001. F.P. and P.N. acknowledge financial support from the Polish Ministry of Science and Higher Education through scholarships for outstanding young scientists. Part of this research was also carried out with the use of instruments available thanks to EC structural funds within the framework of the Center for Preclinical Research and Technology (CePT), POIG No. 02.02.00-17-024/08-00. We would also like to thank Prof. Stefano Piraino (University of Salento) for the valuable discussions on jellyfishes and for lending us the rights to the photo of a Phacellophora camtschatica.

\section{REFERENCES}

(1) Fonseca, A. C.; Serra, A. C.; Coelho, J. F. J. Bioabsorbable Polymers in Cancer Therapy: Latest Developments. EPMA J 2015, 6, 22.

(2) Radovic-Moreno, A. F.; Lu, T. K.; Puscasu, V. A.; Yoon, C. J.; Langer, R.; Farokhzad, O. C. Surface Charge-Switching Polymeric Nanoparticles for Bacterial Cell Wall-Targeted Delivery of Antibiotics. ACS Nano 2012, 6, 4279-4287.

(3) Rosenblum, D.; Joshi, N.; Tao, W.; Karp, J. M.; Peer, D. Progress and Challenges Towards Targeted Delivery of Cancer Therapeutics. Nat. Commun. 2018, 9, 1410.

(4) Spaulding, C. N.; Klein, R. D.; Schreiber, H. L., IV; Janetka, J. W.; Hultgren, S. J. Precision antimicrobial therapeutics: the path of least resistance? NPJ Biofilms Microbiomes 2018, 4, 4.

(5) Zahreddine, H.; Borden, K. L. B. Mechanisms and Insights into Drug Resistance in Cancer. Front. Pharmacol. 2013, 4, 28.

(6) Merino, S.; Martín, C.; Kostarelos, K.; Prato, M.; Vázquez, E. Nanocomposite Hydrogels: 3D Polymer-Nanoparticle Synergies for On-Demand Drug Delivery. ACS Nano 2015, 9, 4686-4697.

(7) Qu, Y.; Chu, B.; Wei, X.; Lei, M.; Hu, D.; Zha, R.; Zhong, L.; Wang, M.; Wang, F.; Qian, Z. Redox/pH Dual-Stimuli Responsive Camptothecin Prodrug Nanogels for "On-Demand" Drug Delivery. J. Controlled Release 2019, 296, 93-106.

(8) Wang, X.; Wang, C.; Zhang, Q.; Cheng, Y. Near Infrared LightResponsive and Injectable Supramolecular Hydrogels For On-Demand Drug Delivery. Chem. Commun. 2016, 52, 978-981.

(9) Pierini, F.; Nakielski, P.; Urbanek, O.; Pawłowska, S.; Lanzi, M.; De Sio, L.; Kowalewski, T. A. Polymer-Based Nanomaterials for Photothermal Therapy: From Light-Responsive to Multifunctional Nanoplatforms for Synergistically Combined Technologies. Biomacromolecules 2018, 19, 4147-4167.

(10) Han, L.-H.; Lai, J. H.; Yu, S.; Yang, F. Dynamic Tissue Engineering Scaffolds with Stimuli-Responsive Macroporosity Formation. Biomaterials 2013, 34, 4251-4258.

(11) Sood, N.; Bhardwaj, A.; Mehta, S.; Mehta, A. Stimuli-Responsive Hydrogels in Drug Delivery and Tissue Engineering. Drug Delivery 2016, 23, 758-780.

(12) Hu, L.; Zhang, Q.; Li, X.; Serpe, M. J. Stimuli-Responsive Polymers for Sensing and Actuation. Mater. Horiz. 2019, 6, 1774-1793.

(13) Koetting, M. C.; Peters, J. T.; Steichen, S. D.; Peppas, N. A. Stimulus-Responsive Hydrogels: Theory, Modern Advances, and Applications. Mater. Sci. Eng. R Rep. 2015, 93, 1-49.

(14) Haq, M. A.; Su, Y.; Wang, D. Mechanical Properties of PNIPAM Based Hydrogels: A Review. Mater. Sci. Eng. C Mater. Biol. Appl. 2017, 70, 842-855.

(15) Liu, T.; Lu, S.; Peng, X.; Jiao, C.; Zhang, J.; Han, M.; Wang, H. Tough, Stimuli-Responsive, and Biocompatible Hydrogels with Very High Water Content. Macromol. Rapid Commun. 2018, 39, 1800474.

(16) Zhang, Y.; Sinha-Ray, S.; Yarin, A. L. Mechanoresponsive Polymer Nanoparticles, Nanofibers and Coatings as Drug Carriers and
Components of Microfluidic Devices. J. Mater. Chem. 2011, 21, 82698281.

(17) Fujishige, S.; Kubota, K.; Ando, I. Phase Transition of Aqueous Solutions of Poly(N-Isopropylacrylamide) and Poly(N-Isopropylmethacrylamide). J. Phys. Chem. 1989, 93, 3311-3313.

(18) Cho, E. C.; Lee, J.; Cho, K. Role of Bound Water and Hydrophobic Interaction in Phase Transition of Poly ( $N$-isopropylacrylamide) Aqueous Solution. Macromolecules 2003, 36, 9929-9934.

(19) Wang, C.; Liu, X.; Wulf, V.; Vázquez-González, M.; Fadeev, M.; Willner, I. DNA-Based Hydrogels Loaded with Au Nanoparticles or Au Nanorods: Thermoresponsive Plasmonic Matrices for Shape-Memory, Self-Healing, Controlled Release, and Mechanical Applications. ACS Nano 2019, 13, 3424-3433.

(20) Pierini, F.; Guglielmelli, A.; Urbanek, O.; Nakielski, P.; Pezzi, L.; Buda, R.; Lanzi, M.; Kowalewski, T. A.; De Sio, L. ThermoplasmonicActivated Hydrogel Based Dynamic Light Attenuator. Adv. Opt. Mater. 2020, 8, 2000324.

(21) De Angelis, B.; Depalo, N.; Petronella, F.; Quintarelli, C.; Curri, M. L.; Pani, R.; Calogero, A.; Locatelli, F.; De Sio, L. StimuliResponsive Nanoparticle-Assisted Immunotherapy: a New Weapon Against Solid Tumours. J. Mater. Chem. B, Mater. Biol. Med. 2020, 8, $1823-1840$

(22) Bleehen, N. M. Hyperthermia in cancer therapy. Br. J. Cancer 1983, 48, 319-319.

(23) Raza, A.; Hayat, U.; Rasheed, T.; Bilal, M.; Iqbal, H. M. N. Smart Materials-Based Near-Infrared Light-Responsive Drug Delivery Systems For Cancer Treatment: A Review. J. Mater. Res. Technol. 2019, 8, 1497-1509.

(24) Wojasiński, M.; Faliszewski, K.; Ciach, T. Electrospinning Production of PLLA Fibrous Scaffolds for Tissue Engineering. Chall. Mod. Technol. 2013, 4, 9-15.

(25) Nakielski, P.; Kowalczyk, T.; Zembrzycki, K.; Kowalewski, T. A. Experimental and Numerical Evaluation of Drug Release from Nanofiber Mats to Brain Tissue. J. Biomed. Mater. Res. 2015, 103, 282-291.

(26) Metwally, S.; Karbowniczek, J. E.; Szewczyk, P. K.; Marzec, M. M.; Gruszczyński, A.; Bernasik, A.; Stachewicz, U. Single-Step Approach to Tailor Surface Chemistry and Potential on Electrospun PCL Fibers for Tissue Engineering Application. Adv. Mater. Interfaces 2019, 6, 1801211.

(27) Nakielski, P.; Pawłowska, S.; Pierini, F.; Liwińska, W.; Hejduk, P.; Zembrzycki, K.; Zabost, E.; Kowalewski, T. A. Hydrogel Nanofilaments via Core-Shell Electrospinning. PLoS One 2015, 10, No. e0129816.

(28) Lavielle, N.; Hébraud, A.; Thöny-Meyer, L.; Rossi, R. M.; Schlatter, G. 3D Composite Assemblies of Microparticles and Nanofibers for Tailored Wettability and Controlled Drug Delivery. Macromol. Mater. Eng. 2017, 302, 1600458.

(29) Chou, S.-F.; Carson, D.; Woodrow, K. A. Current Strategies for Sustaining Drug Release from Electrospun Nanofibers. J. Controlled Release 2015, 220, 584-591.

(30) D’Amato, A. R.; Schaub, N. J.; Cardenas, J. M.; Fiumara, A. S.; Troiano, P. M.; Fischetti, A.; Gilbert, R. J. Removal of Retained Electrospinning Solvent Prolongs Drug Release from Electrospun PLLA Fibers. Polymer 2017, 123, 121-127.

(31) Nguyen, T. T. T.; Ghosh, C.; Hwang, S.-G.; Chanunpanich, N.; Park, J. S. Porous Core/Sheath Composite Nanofibers Fabricated by Coaxial Electrospinning as a Potential Mat For Drug Release System. Int. J. Pharm. 2012, 439, 296-306.

(32) Torres-Martinez, E. J.; Cornejo Bravo, J. M.; Serrano Medina, A.; Pérez González, G. L.; Villarreal Gómez, L. J. A Summary of Electrospun Nanofibers as Drug Delivery System: Drugs Loaded and Biopolymers Used as Matrices. Curr. Drug Delivery 2018, 15, 13601374.

(33) Huang, X.; Brazel, C. S. On the Importance and Mechanisms of Burst Release in Matrix-Controlled Drug Delivery Systems. J. Controlled Release 2001, 73, 121-136.

(34) Zhu, J.; Wang, X.; He, C.; Wang, H. Mechanical Properties, Anisotropic Swelling Behaviours and Structures of Jellyfish Mesogloea. J Mech Behav Biomed Mater 2012, 6, 63-73. 
(35) Wei, H.; Shi, N.; Zhang, J.; Guan, Y.; Zhang, J.; Wan, X. pHResponsive Inorganic-Organic Hybrid Supramolecular Hydrogels with Jellyfish-Like Switchable Chromic Luminescence. Chem. Commun. 2014, 50, 9333-9335.

(36) Peng, X.; He, C.; Liu, J.; Wang, H. Biomimetic Jellyfish-Like PVA/Graphene Oxide Nanocomposite Hydrogels with Anisotropic and $\mathrm{pH}$-Responsive Mechanical Properties. J. Mater. Sci. 2016, 51, 5901-5911.

(37) Jabbari, E.; Kim, D.; Lee, L. P.; Ghaemmaghami, A.; Khademhosseini, A. Handbook of Biomimetics and Bioinspiration: Biologically-Driven Engineering of Materials, Processes, Devices, and Systems (In 3 Volumes); World scientific series in nanoscience and nanotechnology; World Scientific: 2014; Vol. 9.

(38) Cao, J.; Chen, Z.; Chi, J.; Sun, Y.; Sun, Y. Recent Progress in Synergistic Chemotherapy and Phototherapy by Targeted Drug Delivery Systems for Cancer Treatment. Artif. Cells Nanomed. Biotechnol. 2018, 46, 817-830.

(39) Martinsson, E.; Shahjamali, M. M.; Large, N.; Zaraee, N.; Zhou, Y.; Schatz, G. C.; Mirkin, C. A.; Aili, D. Influence of Surfactant Bilayers on The Refractive Index Sensitivity and Catalytic Properties of Anisotropic Gold Nanoparticles. Small 2016, 12, 330-342.

(40) Huang, X.; El-Sayed, M. A. Gold Nanoparticles: Optical Properties and Implementations in Cancer Diagnosis and Photothermal Therapy. J. Adv. Res. 2010, 1, 13-28.

(41) Bellotti, E.; Fedorchak, M. V.; Velankar, S.; Little, S. R. Tuning of Thermoresponsive PNIPAAm Hydrogels for the Topical Retention of Controlled Release Ocular Therapeutics. J. Mater. Chem. B, Mater. Biol. Med. 2019, 7, 1276-1283.

(42) Guo, H.; Mussault, C.; Marcellan, A.; Hourdet, D.; Sanson, N. Hydrogels with Dual Thermoresponsive Mechanical Performance. Macromol. Rapid Commun. 2017, 38, 1700287.

(43) Ghugare, S. V.; Chiessi, E.; Telling, M. T. F.; Deriu, A.; Gerelli, Y.; Wuttke, J.; Paradossi, G. Structure and Dynamics of a Thermoresponsive Microgel Around its Volume Phase Transition Temperature. J. Phys. Chem. B 2010, 114, 10285-10293.

(44) Futscher, M. H.; Philipp, M.; Müller-Buschbaum, P.; Schulte, A. The Role of Backbone Hydration of Poly(N-isopropyl acrylamide) Across the Volume Phase Transition Compared to its Monomer. Sci. Rep. 2017, 7, 17012.

(45) Kokufuta, M. K.; Sato, S.; Kokufuta, E. LCST Behavior of Copolymers of $\mathrm{N}$-isopropylacrylamide and $\mathrm{N}$-isopropylmethacrylamide in Water. Colloid Polym. Sci. 2012, 290, 1671-1681.

(46) Starovoytova, L.; Spěváček, J.; Ilavský, M. 1H NMR Study of Temperature-Induced Phase Transitions in $\mathrm{D}_{2} \mathrm{O}$ Solutions of Poly $(\mathrm{N}$ isopropylmethacrylamide)/Poly(N-isopropylacrylamide) mixtures and Random Copolymers. Polymer 2005, 46, 677-683.

(47) Kratz, K.; Hellweg, T.; Eimer, W. Structural Changes in PNIPAM Microgel Particles as Seen by SANS, DLS, and EM Techniques. Polymer 2001, 42, 6631-6639.

(48) Ikeda-Fukazawa, T.; Ikeda, N.; Tabata, M.; Hattori, M.; Aizawa, M.; Yunoki, S.; Sekine, Y. Effects of Crosslinker Density on The Polymer Network Structure in Poly-N,N-Dimethylacrylamide Hydrogels. J. Polym. Sci., Part B: Polym. Phys. 2013, 51, 1017-1027.

(49) Liu, S.; Wang, L.; Lin, M.; Wang, D.; Song, Z.; Li, S.; Ge, R.; Zhang, X.; Liu, Y.; Li, Z.; Sun, H.; Yang, B.; Zhang, H. Cu(II)-Doped Polydopamine-Coated Gold Nanorods for Tumor Theranostics. ACS Appl. Mater. Interfaces 2017, 9, 44293-44306.

(50) Zeng, J.; Yang, L.; Liang, Q.; Zhang, X.; Guan, H.; Xu, X.; Chen, $\mathrm{X}$.; Jing, X. Influence of the Drug Compatibility with Polymer Solution on The Release Kinetics of Electrospun Fiber Formulation. J. Controlled Release 2005, 105, 43-51.

(51) Kenawy, E.-R.; Bowlin, G. L.; Mansfield, K.; Layman, J.; Simpson, D. G.; Sanders, E. H.; Wnek, G. E. Release of Tetracycline Hydrochloride from Electrospun Poly(Ethylene-Co-Vinylacetate), Poly(Lactic Acid), and a Blend. J. Controlled Release 2002, 81, 57-64. (52) Ball, C.; Krogstad, E.; Chaowanachan, T.; Woodrow, K. A. DrugEluting Fibers for HIV-1 Inhibition and Contraception. PLoS One 2012, 7, No. e49792.
(53) Yixiang, D.; Yong, T.; Liao, S.; Chan, C. K.; Ramakrishna, S. Degradation of Electrospun Nanofiber Scaffold by Short Wave Length Ultraviolet Radiation Treatment and its Potential Applications in Tissue Engineering. Tissue Eng. Part A 2008, 14, 1321-1329.

(54) Valente, T. A. M.; Silva, D. M.; Gomes, P. S.; Fernandes, M. H.; Santos, J. D.; Sencadas, V. Effect of Sterilization Methods on Electrospun Poly(lactic acid) (PLA) Fiber Alignment for Biomedical Applications. ACS Appl. Mater. Interfaces 2016, 8, 3241-3249.

(55) Kim, M.; Lee, J.-H.; Nam, J.-M. Plasmonic Photothermal Nanoparticles for Biomedical Applications. Adv Sci (Weinh) 2019, 6, 1900471.

(56) De Sio, L.; Placido, T.; Comparelli, R.; Lucia Curri, M.; Striccoli, M.; Tabiryan, N.; Bunning, T. J. Next-Generation Thermo-Plasmonic Technologies and Plasmonic Nanoparticles in Optoelectronics. Prog. Quantum Electron. 2015, 41, 23-70.

(57) Srikar, R.; Yarin, A. L.; Megaridis, C. M.; Bazilevsky, A. V.; Kelley, E. Desorption-Limited Mechanism of Release from Polymer Nanofibers. Langmuir 2008, 24, 965-974.

(58) Gandhi, M.; Srikar, R.; Yarin, A. L.; Megaridis, C. M.; Gemeinhart, R. A. Mechanistic Examination of Protein Release from Polymer Nanofibers. Mol. Pharmaceutics 2009, 6, 641-647.

(59) Khansari, S.; Duzyer, S.; Sinha-Ray, S.; Hockenberger, A.; Yarin, A. L.; Pourdeyhimi, B. Two-Stage Desorption-Controlled Release of Fluorescent Dye and Vitamin from Solution-Blown and Electrospun Nanofiber Mats Containing Porogens. Mol. Pharmaceutics 2013, 10, 4509-4526.

(60) Naik, A.; Kalia, Y. N.; Guy, R. H. Transdermal Drug Delivery: Overcoming the Skin's Barrier Function. Pharm. Sci. Technol. Today 2000, 3, 318-326.

(61) Mofidfar, M.; O’Farrell, L.; Prausnitz, M. R. Pharmaceutical Jewelry: Earring Patch for Transdermal Delivery of Contraceptive Hormone. J. Controlled Release 2019, 301, 140-145.

(62) Hsiao, C.-W.; Chen, H.-L.; Liao, Z.-X.; Sureshbabu, R.; Hsiao, H.-C.; Lin, S.-J.; Chang, Y.; Sung, H.-W. Effective Photothermal Killing of Pathogenic Bacteria by Using Spatially Tunable Colloidal Gels with Nano-Localized Heating Sources. Adv. Funct. Mater. 2015, 25, 721728.

(63) Xu, J.-W.; Yao, K.; Xu, Z.-K. Nanomaterials with A Photothermal Effect for Antibacterial Activities: An Overview. Nanoscale 2019, 11, $8680-8691$. 